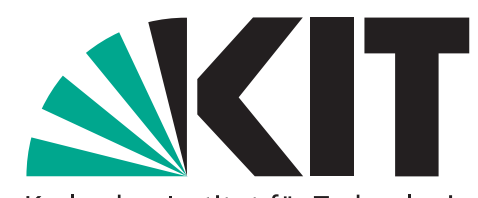

Karlsruher Institut für Technologie

\title{
Tempered stable and tempered infinitely divisible GARCH models
}

by Young Shin Kim, Svetlozar T. Rachev, Michele Leonardo Bianchi, Frank J. Fabozzi

No. 28 | MAY 2011

\section{WORKING PAPER SERIES IN ECONOMICS}

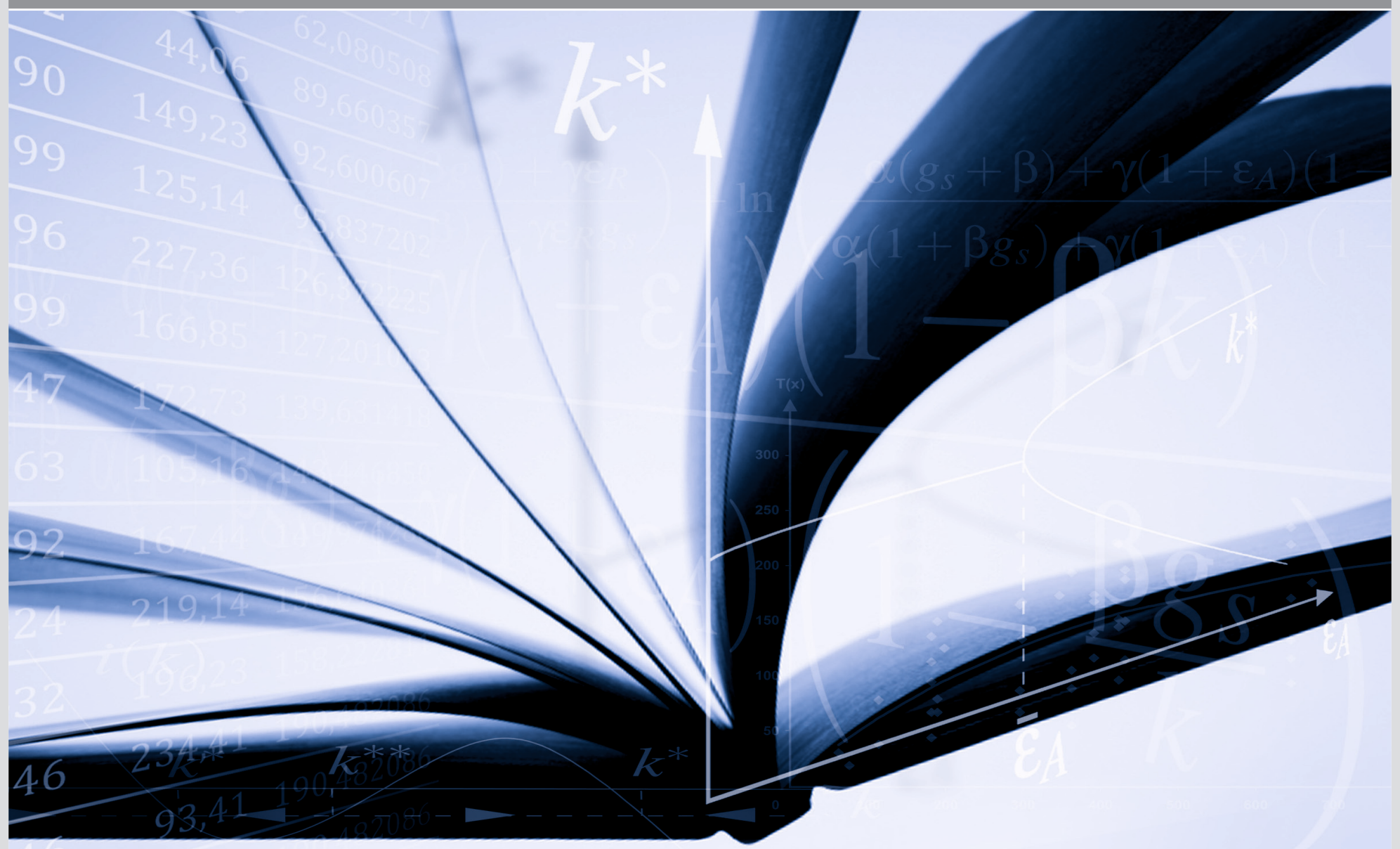




\section{Impressum}

Karlsruher Institut für Technologie (KIT)

Fakultät für Wirtschaftswissenschaften

Institut für Wirtschaftspolitik und Wirtschaftsforschung (IWW)

Institut für Wirtschaftstheorie und Statistik (ETS)

Schlossbezirk 12

76131 Karlsruhe

KIT - Universität des Landes Baden-Württemberg und nationales Forschungszentrum in der Helmholtz-Gemeinschaft

Working Paper Series in Economics

No. 28, May 2011

ISSN 2190-9806

econpapers.wiwi.kit.edu 


\title{
Tempered stable and tempered infinitely divisible GARCH models
}

\author{
Young Shin Kim \\ School of Economics and Business Engineering, University of Karlsruhe and KIT
}

Svetlozar T. Rachev

School of Economics and Business Engineering, University of Karlsruhe and KIT, and Department of Statistics and Applied Probability, University of California, Santa Barbara, and FinAnalytica INC

\author{
Michele Leonardo Bianchi \\ University of Bergamo \\ Frank J. Fabozzi \\ Yale School of Management
}

\begin{abstract}
In this paper, we introduce a new GARCH model with an infinitely divisible distributed innovation, referred to as the rapidly decreasing tempered stable (RDTS) GARCH model. This model allows the description of some stylized empirical facts observed for stock and index returns, such as volatility clustering, the non-zero skewness and excess kurtosis for the residual distribution. Furthermore, we review the classical tempered stable (CTS) GARCH model, which has similar statistical properties. By considering a proper density transformation between infinitely divisible random variables, these GARCH models allow to find the risk-neutral price process, and hence they can be applied to option pricing. We propose algorithms to generate scenario based on GARCH models with CTS and RDTS innovation. To investigate the performance of these GARCH models, we report a parameters estimation for Dow Jones Industrial Average (DJIA) index and stocks included in this index, and furthermore to demonstrate their advantages, we calculate option prices based on these models. It should be noted that only historical data on the underlying asset and on the riskfree rate are taken into account to evaluate option prices.
\end{abstract}

Keywords: tempered infinitely divisible distribution, tempered stable distribution, rapidly decreasing tempered stable distribution, GARCH model option pricing.

Svetlozar T. Rachev gratefully acknowledges research support by grants from Division of Mathematical, Life and Physical Sciences, College of Letters and Science, University of California, Santa Barbara, the Deutschen Forschungsgemeinschaft and the Deutscher Akademischer Austausch Dienst. The authors thank Prof. Gennady Samorodnitsky for his help in formulating the problem of TID distributions and for his fruitful comments and suggestions. 


\section{Introduction}

The autoregressive conditional heteroskedastic (ARCH) and the generalized ARCH (GARCH) models introduced by Engle (1982) and Bollerslev (1986), respectively, and applied to option pricing by Duan (1995), have become a standard framework to explain the volatility clustering of return processes and volatility smile effect of option prices. However, empirical studies based on GARCH models show that the hypothesis that the distribution of residuals is normally distributed is often rejected (see Duan (1999), and Menn and Rachev (2005a, 2005b)). Duan et al. (2006) enhanced the classical GARCH model by adding jumps to the innovation process. Subsequently, Menn and Rachev (2005a, 2005b) introduced both an enhanced GARCH and a nonlinear GARCH model (NGARCH) with innovations which follow the smoothly truncated stable (STS) distribution. Recently, the tempered stable distributions were applied to modeling the residual distribution. For example Kim et al. (2008a,2008c) used the tempered stable distributions for fitting residuals of the GARCH model. However, since the convexity correction, which is defined by the log Laplace transform of the innovation distribution, is defined only on a bounded interval, the variance process is artificially restricted.

In this paper, we focus on two different distributional assumptions, the classical tempered stable (CTS) and the rapidly decreasing tempered stable (RDTS). The former belongs to the class proposed by Rosiński (2007) and has been already applied to option pricing with volatility clustering by Kim et al. (2008a), the latter belongs to the class proposed by Bianchi et al. (2008).

The first objective of the paper is to present this new infinitely divisible (ID) distribution referred to as the RDTS distribution, and to study its mathematical properties. The RDTS distribution is obtained by taking an $\alpha$-stable law and multiplying the Lévy measure by a moment-generating function of a normal distribution onto each half of the real axis. It has asymmetric properties and fatter tails than the normal distribution. Moreover, its Laplace transform is defined on the entire real line. By following the approach used in Kim et al. (2008a), we review an asset price model based on the GARCH model with 
CTS distributed innovation, introduce a similar model with RDTS distributed innovation, and compare it with the normal-GARCH case. These non-normal models explain the time-varying property of volatility in asset returns, and describe properties of the empirical residual distribution which cannot be described by the normal distribution including skewness and fat-tail properties. Furthermore, a large scale empirical analysis is considered on the Dow Jones Industrial Average (DJIA) index and stocks included in this index, in order to assess the goodness of fit.

The second objective of the paper is to test the option pricing performance of this approach based on non-normal distributions. Recently, a general idea has been that for the purpose of option valuation, parameters estimated from option prices are preferable to parameters estimated from the underlying returns, see Chernov and Ghysels (2000). Alternatively, the most recent results are based on a different approach. Both historical asset prices and option prices are considered to assess the model performance. Parametric models by Christoffersen et al. (2008), Kim et al. (2008a), Stentoft (2008), and a nonparametric one by Barone-Adesi et al. (2008) have been proposed by connecting the statistical with the risk-neutral measure. Instead of imposing conditions on preferences of investors or the Esscher transform as in Christoffersen et al. (2008), by using a density transformation between ID random variables, we can then develop a method for pricing options based on these GARCH models, see also Kim et al. (2008a,2008c). It should be noted that only historical data on the underlying asset and on the risk-free rate are considered in obtaining the parameters to be used in option valuation. Instead, to consider a trader approach, in which one wants to estimate parameters by using only option prices, we follow the so called fundamental approach, that is we calculate option prices by using parameters estimated by fitting the underlying asset process together with a suitable change of measure. Pricing errors on DJIA European call options (DJX) will be computed, in order to analyze the effect of conditional leptokurtosis and skewness on option pricing.

The remainder of this paper is organized as follows. Section 2 reviews the classical 
tempered stable distribution. The RDTS distribution and its mathematical properties are presented in Section 3. The GARCH model with ID innovations and its CTS and RDTS subclasses are discussed in Section 4. Simulation algorithms for the GARCH models are given in Section 5. The empirical results are reported in Section 6. Section 7 summarizes the principal conclusions of the paper and the appendix contains the proofs of the main theoretical results.

\section{Classical tempered stable distribution}

Before introducing the RDTS distribution, let us review the CTS distribution. This distribution has been studied under different names including: the truncated Lévy flight by Koponen (1995), the tempered stable by both Barndorff-Nielsen and Shephard (2001) and Cont and Tankov (2004), the KoBoL distribution by Boyarchenko and Levendorskil (2000), and the CGMY by Carr et al. (2002). The KR distribution of Kim et al. (2008b) is an extension of the CTS distribution. Rosiński (2007) generalized the CTS distribution referring to it as the tempered stable distribution.

The CTS distribution is defined as follows:

Definition 2.1. An infinitely divisible random variable $X$ is said to follow the CTS distribution if its Lévy triplet $\left(\sigma^{2}, \nu, \gamma\right)$ is given by $\sigma=0$,

$$
\nu(d x)=\left(C_{+} e^{-\lambda_{+} x} 1_{x>0}+C_{-} e^{-\lambda_{-}|x|} 1_{x<0}\right) \frac{d x}{|x|^{\alpha+1}},
$$

and

$$
\gamma=m-\int_{|x|>1} x \nu(d x),
$$

where $C_{+}, C_{-}, \lambda_{+}, \lambda_{-}>0, \alpha \in(0,2)$ and $m \in \mathbb{R}$, and we denote $X \sim \operatorname{CTS}\left(\alpha, C_{+}\right.$, $\left.C_{-}, \lambda_{+}, \lambda_{-}, m\right)$. A Lévy process induced from the CTS distribution is called a CTS process with parameters $\left(\alpha, C_{+}, C_{-}, \lambda_{+}, \lambda_{-}, m\right)$. 
The characteristic function of $X \sim \operatorname{CTS}\left(\alpha, C_{+}, C_{-}, \lambda_{+}, \lambda_{-}, m\right)$ is given by

$$
\begin{aligned}
\phi\left(u ; \alpha, C_{+}, C_{-}, \lambda_{+}, \lambda_{-}, m\right)=\exp (i u m & -i u \Gamma(1-\alpha)\left(C_{+} \lambda_{+}^{\alpha-1}-C_{-} \lambda_{-}^{\alpha-1}\right) \\
& +C_{+} \Gamma(-\alpha)\left(\left(\lambda_{+}-i u\right)^{\alpha}-\lambda_{+}{ }^{\alpha}\right) \\
& \left.\left.+C_{-} \Gamma(-\alpha)\left(\left(\lambda_{-}+i u\right)^{\alpha}-\lambda_{-}{ }^{\alpha}\right)\right)\right) .
\end{aligned}
$$

Moreover, $\phi$ can be extended via analytic continuation to the region $\{z \in \mathbb{C}: \operatorname{Im}(z) \in$ $\left.\left[-\lambda_{-}, \lambda_{+}\right]\right\}$. The proof can be found in Carr et al. (2002) and Cont and Tankov (2004). Using the characteristic function, we can obtain cumulants

$$
c_{n}(X):=\left.\frac{1}{i^{n}} \frac{d^{n}}{d u^{n}} \log E\left[e^{i u X}\right]\right|_{u=0}
$$

of the CTS distributed random variable $X$ such that

$$
\begin{array}{ll}
c_{1}(X)=m, & \text { for } n=1 \\
c_{n}(X)=\Gamma(n-\alpha)\left(C_{+} \lambda_{+}^{\alpha-n}+(-1)^{n} C_{-} \lambda_{-}^{\alpha-n}\right), & \text { for } n=2,3, \cdots .
\end{array}
$$

If we substitute

$$
C=C_{+}=C_{-}=\left(\Gamma(2-\alpha)\left(\lambda_{+}{ }^{\alpha-2}+\lambda_{-}{ }^{\alpha-2}\right)\right)^{-1}
$$

then $X \sim \operatorname{CTS}\left(\alpha, C, C, \lambda_{+}, \lambda_{-}, 0\right)$ has zero mean and unit variance. In this case, $X$ is called the standard CTS distribution with parameters $\left(\alpha, \lambda_{+}, \lambda_{-}\right)$and denoted by $X \sim \operatorname{stdCTS}\left(\alpha, \lambda_{+}, \lambda_{-}\right)$. The $\log$-Laplace transform $\log E[\exp (u X)]$ of the random variable $X \sim \operatorname{stdCTS}\left(\alpha, \lambda_{+}, \lambda_{-}\right)$is denoted by $L_{C T S}\left(u ; \alpha, \lambda_{+}, \lambda_{-}\right)$. The function $L_{C T S}\left(u ; \alpha, \lambda_{+}, \lambda_{-}\right)$is defined on $u \in\left[-\lambda_{-}, \lambda_{+}\right]$and we can obtain

$$
\begin{aligned}
& L_{C T S}\left(u ; \alpha, \lambda_{+}, \lambda_{-}\right) \\
& =\frac{\left(\lambda_{+}-u\right)^{\alpha}-\lambda_{+}{ }^{\alpha}+\left(\lambda_{-}+u\right)^{\alpha}-\lambda_{-}{ }^{\alpha}}{\alpha(\alpha-1)\left({\lambda_{+}}^{\alpha-2}+\lambda_{-}{ }^{\alpha-2}\right)}-\frac{u\left(\lambda_{+}{ }^{\alpha-1}-\lambda_{-}{ }^{\alpha-1}\right)}{(1-\alpha)\left({\lambda_{+}}^{\alpha-2}+\lambda_{-}{ }^{\alpha-2}\right)}
\end{aligned}
$$


by the characteristic function (2.1).

We can make use of the following proposition proven in Kim and Lee (2006) to find an equivalent measure for CTS processes.

Proposition 2.2. Suppose $\left(X_{t}\right)_{t \in[0, T]}$ is the CTS process with parameters $\left(\alpha, C_{+}, C_{-}\right.$, $\left.\lambda_{+}, \lambda_{-}, m\right)$ under $\mathbb{P}$ and the CTS process with parameters $\left(\tilde{\alpha}, \tilde{C}_{+}, \tilde{C}_{-}, \tilde{\lambda}_{+}, \tilde{\lambda}_{-}, \tilde{m}\right)$ under $\mathbb{Q}$. Then $\left.\mathbb{P}\right|_{\mathcal{F}_{t}}$ and $\left.\mathbb{Q}\right|_{\mathcal{F}_{t}}$ are equivalent for all $t>0$ if and only if $\alpha=\tilde{\alpha}, C_{+}=\tilde{C}_{+}$, $C_{-}=\tilde{C}_{-}$, and

$$
\tilde{m}-m=\Gamma(1-\alpha)\left(C_{+}\left(\tilde{\lambda}_{+}^{\alpha-1}-\lambda_{+}^{\alpha-1}\right)-C_{-}\left(\tilde{\lambda}_{-}^{\alpha-1}-\lambda_{-}^{\alpha-1}\right)\right) .
$$

When $\mathbb{P}$ and $\mathbb{Q}$ are equivalent, the Radon-Nikodym derivative is $\left.\frac{d \mathbf{Q}}{d \mathbf{P}}\right|_{\mathcal{F}_{t}}=e^{U_{t}}$ where $\left(U_{t}, \mathbf{P}\right)$ is a Lévy process with Lévy triplets $\left(\sigma_{U}^{2}, \nu_{U}, \gamma_{U}\right)$ given by

$$
\sigma_{U}^{2}=0, \nu_{U}=\nu \circ \psi^{-1}, \gamma_{U}=-\int_{-\infty}^{\infty}\left(e^{y}-1-y 1_{|y| \leq 1}\right)\left(\nu \circ \psi^{-1}\right)(d y)
$$

where $\psi(x)=\left(\lambda_{+}-\tilde{\lambda}_{+}\right) x 1_{x>0}-\left(\lambda_{-}-\tilde{\lambda}_{-}\right) x 1_{x<0}$.

Applying Proposition 2.2 to CTS distributed random variables, we obtain the following corollary.

Corollary 2.3. (a) Let $X \sim \operatorname{CTS}\left(\alpha, C_{+}, C_{-}, \lambda_{+}, \lambda_{-}, m\right)$ under a measure $\mathbf{P}$, and $X \sim \operatorname{CTS}\left(\tilde{\alpha}, \tilde{C}_{+}, \tilde{C}_{-}, \tilde{\lambda}_{+}, \tilde{\lambda}_{-}, \tilde{m}\right)$ under a measure $\mathbf{Q}$. Then $\mathbf{P}$ and $\mathbf{Q}$ are equivalent if and only if $\alpha=\tilde{\alpha}, C_{+}=\tilde{C}_{+}, C_{-}=\tilde{C}_{-}$, and

$$
\tilde{m}-m=\Gamma(1-\alpha)\left(C_{+} \tilde{\lambda}_{+}^{\alpha-1}-C_{-} \tilde{\lambda}_{-}^{\alpha-1}-C_{+} \lambda_{+}^{\alpha-1}+C_{-} \lambda_{-}^{\alpha-1}\right) .
$$

(b) Let $X \sim \operatorname{stdCTS}\left(\alpha, \lambda_{+}, \lambda_{-}\right)$under a measure $\mathbf{P}$, and $(X+k) \sim \operatorname{stdCTS}\left(\tilde{\alpha}, \tilde{\lambda}_{+}, \tilde{\lambda}_{-}\right)$ 
under a measure $\mathbf{Q}$ for a constant $k \in \mathbb{R}$. Then $\mathbf{P}$ and $\mathbf{Q}$ are equivalent if and only if

$$
\left(\begin{array}{l}
\alpha=\tilde{\alpha} \\
\lambda_{+}{ }^{\alpha-2}+\lambda_{-}{ }^{\alpha-2}=\tilde{\lambda}_{+}^{\alpha-2}+\tilde{\lambda}_{-}^{\alpha-2} \\
k=\frac{\lambda_{+}{ }^{\alpha-1}-\lambda_{-}{ }^{\alpha-1}-\tilde{\lambda}_{+}^{\alpha-1}+\tilde{\lambda}_{-}^{\alpha-1}}{(1-\alpha)\left({\lambda_{+}}^{\alpha-2}+\lambda_{-}{ }^{\alpha-2}\right)} .
\end{array}\right.
$$

\subsection{Simulation of the CTS distribution}

CTS distributed random numbers can be generated using the subordination method developed by Poirot and Tankov (2006). Here, we will apply the series representation presented by Rosiński (2007) to the CTS distribution instead of the subordination method, see also Asmussen and Glynn (2007).

Consider $\alpha \in(0,2), C>0$, and $\lambda_{+}, \lambda_{-}>0$. Let $\left\{v_{j}\right\}$ be an independent and identically distributed (i.i.d.) sequence of random variables in $\left\{\lambda_{+}, \lambda_{-}\right\}$with $P\left(v_{j}=\right.$ $\left.\lambda_{+}\right)=P\left(v_{j}=-\lambda_{-}\right)=1 / 2$. Let $\left\{u_{j}\right\}$ be an i.i.d. sequence of uniform random variables on $(0,1)$ and let $\left\{e_{j}\right\}$ and $\left\{e_{j}^{\prime}\right\}$ be i.i.d. sequences of exponential random variables with parameters 1. Furthermore, we assume that $\left\{v_{j}\right\},\left\{u_{j}\right\},\left\{e_{j}\right\}$, and $\left\{e_{j}^{\prime}\right\}$ are independent. We consider $\gamma_{j}=e_{1}^{\prime}+\ldots+e_{j}^{\prime}$ and, by definition of $\left\{e_{j}^{\prime}\right\},\left\{\gamma_{j}\right\}$ is a Poisson point process on $(0, \infty)$ with Lebesgue intensity measure. Based on these assumption, we can prove the next theorem.

Theorem 2.4. Suppose that all the above assumptions are fulfilled. If $\alpha \in(0,2) \backslash\{1\}$, the series

$$
S=\sum_{j=1}^{\infty}\left(\left(\frac{\alpha \gamma_{j}}{C}\right)^{-1 / \alpha} \wedge e_{j} u_{j}^{1 / \alpha}\left|v_{j}\right|^{-1}\right) \frac{v_{j}}{\left|v_{j}\right|}-\Gamma(1-\alpha) C\left(\lambda_{+}^{\alpha-1}-\lambda_{-}^{\alpha-1}\right)
$$

converges a.s. Furthermore, we have that $S \sim C T S\left(\alpha, C, C, \lambda_{+}, \lambda_{-}, 0\right)$

If $\alpha=1$, the series

$$
S=\sum_{j=1}^{\infty}\left(\left(\frac{\alpha \gamma_{j}}{C}\right)^{-1} \wedge e_{j} u_{j}\left|v_{j}\right|^{-1}\right) \frac{v_{j}}{\left|v_{j}\right|}-C \log \left(\frac{\lambda_{+}}{\lambda_{-}}\right)
$$


converges a.s. and we have $S \sim C T S\left(1, C, C, \lambda_{+}, \lambda_{-}, 0\right)$.

Proof. This is a particular case of Theorem 5.1 in Rosiński (2007).

\section{Rapidly decreasing tempered stable distribution}

In this section, we present an ID distribution which we refer to as the RDTS distribution. This distribution is defined as follows:

Definition 3.1. Let $m \in \mathbb{R}, C_{+}, C_{-}, \lambda_{+}, \lambda_{-}>0, \alpha \in(0,2)$, and $\alpha \neq 1$. An infinitely divisible distribution is called a RDTS distribution with parameter $\left(\alpha, C_{+}, C_{-}, \lambda_{+}, \lambda_{-}\right.$, $m)$ if its Lévy triplet $\left(\sigma^{2}, \nu, \gamma\right)$ is given by $\sigma=0$,

$$
\nu(d x)=\left(C_{+} e^{-\lambda_{+}^{2} x^{2} / 2} 1_{x>0}+C_{-} e^{-\lambda_{-}^{2}|x|^{2} / 2} 1_{x<0}\right) \frac{d x}{|x|^{\alpha+1}},
$$

and

$$
\gamma=m-\int_{|x|>1} x \nu(d x)
$$

If a random variable $X$ follows the RDTS distribution, then we denote $X \sim \operatorname{RDTS}(\alpha$, $\left.C_{+}, C_{-}, \lambda_{+}, \lambda_{-}, m\right)$.

Remark 3.2. RDTS distributions are not included in the generalized class of tempered stable distributions by Rosinski (2007), but included in the class of the tempered infinitely divisible distribution (Bianchi et al. (2008)).

The characteristic function of the RDTS distribution is found in the following proposition and its proof is presented in Appendix A.

Proposition 3.3. Let

$$
\begin{aligned}
G(x ; \alpha, \lambda): & =2^{-\frac{\alpha}{2}-1} \lambda^{\alpha} \Gamma\left(-\frac{\alpha}{2}\right)\left(M\left(-\frac{\alpha}{2}, \frac{1}{2} ; \frac{x^{2}}{2 \lambda^{2}}\right)-1\right) \\
& +2^{-\frac{\alpha}{2}-\frac{1}{2}} \lambda^{\alpha-1} x \Gamma\left(\frac{1-\alpha}{2}\right)\left(M\left(\frac{1-\alpha}{2}, \frac{3}{2} ; \frac{x^{2}}{2 \lambda^{2}}\right)-1\right)
\end{aligned}
$$


where $M$ is the confluent hypergeometric function ${ }^{1}$. The characteristic function of the RDTS distribution with parameter $\left(\alpha, C_{+}, C_{-}, \lambda_{+}, \lambda_{-}, m\right)$ becomes

$$
\phi(u)=\exp \left(i u m+C_{+} G\left(i u ; \alpha, \lambda_{+}\right)+C_{-} G\left(-i u ; \alpha, \lambda_{-}\right)\right)
$$

for some $m \in \mathbb{R}$. Moreover, $\phi(u)$ is expandable to an entire function on $\mathbb{C}$.

Although the Laplace transform of the CTS distribution is defined on a bounded interval, in the case of the RDTS distribution the Laplace transform is defined on the entire real line.

Proposition 3.4. Let $X \sim \operatorname{RDTS}\left(\alpha, C_{+}, C_{-}, \lambda_{+}, \lambda_{-}, m\right)$. Then the Laplace transform $E\left[e^{\theta X}\right]<\infty$ for all $\theta \in \mathbb{R}$. Moreover, the explicit formula of the Laplace transform is given by

$$
E\left[e^{\theta X}\right]=\exp \left(\theta m+C_{+} G\left(\theta ; \alpha, \lambda_{+}\right)+C_{-} G\left(-\theta ; \alpha, \lambda_{-}\right)\right)
$$

Using the characteristic function (3.2), we can get cumulants of the RDTS distribution.

Proposition 3.5. The cumulants of $X \sim \operatorname{RDTS}\left(\alpha, C_{+}, C_{-}, \lambda_{+}, \lambda_{-}, m\right)$ are given by $c_{1}(X)=m$ and

$$
c_{n}(X)=2^{\frac{n-\alpha-2}{2}} \Gamma\left(\frac{n-\alpha}{2}\right)\left(C_{+} \lambda_{+}^{\alpha-n}+(-1)^{n} C_{-} \lambda_{-}^{\alpha-n}\right)
$$

for $n=2,3, \cdots$.

Proof. Since we have

$$
G\left( \pm i u ; \alpha, \lambda_{ \pm}\right)=\sum_{n=2}^{\infty} \frac{1}{n !}( \pm i u)^{n} \frac{1}{2}\left(\frac{\lambda_{ \pm}}{\sqrt{2}}\right)^{\alpha-n} \Gamma\left(\frac{n-\alpha}{2}\right)
$$

we deduce

$$
\left.\frac{1}{i} \frac{d}{d u} G\left( \pm i u ; \alpha, \lambda_{ \pm}\right)\right|_{u=0}=0
$$


and

$$
\left.\frac{1}{i^{n}} \frac{d^{n}}{d u^{n}} G\left( \pm i u ; \alpha, \lambda_{ \pm}\right)\right|_{u=0}=\frac{1}{2}\left(\frac{\lambda_{ \pm}}{\sqrt{2}}\right)^{\alpha-n} \Gamma\left(\frac{n-\alpha}{2}\right)(-i)^{n}
$$

if $n=2,3, \cdots$. Hence we obtain the formula (3.3).

Moreover, we obtain the mean, variance, skewness, and excess kurtosis using the cumulants as given below:

$$
\begin{aligned}
E[X] & =c_{1}(X)=m \\
\operatorname{Var}(X) & =c_{2}(X)=2^{-\frac{\alpha}{2}} \Gamma\left(1-\frac{\alpha}{2}\right)\left(C_{+} \lambda_{+}^{\alpha-2}+C_{-} \lambda_{-}^{\alpha-2}\right) \\
s(X) & =\frac{c_{3}(X)}{c_{2}(X)^{\frac{3}{2}}}=2^{\frac{\alpha}{4}+\frac{1}{2}} \frac{\Gamma\left(\frac{3-\alpha}{2}\right)\left(C_{+} \lambda_{+}^{\alpha-3}-C_{-} \lambda_{-}^{\alpha-3}\right)}{\left(\Gamma\left(1-\frac{\alpha}{2}\right)\left(C_{+} \lambda_{+}^{\alpha-2}+C_{-} \lambda_{-}^{\alpha-2}\right)\right)^{\frac{3}{2}}} \\
k(X) & =\frac{c_{4}(X)}{c_{2}(X)^{2}}=2^{\frac{\alpha}{2}+1} \frac{\Gamma\left(\frac{4-\alpha}{2}\right)\left(C_{+} \lambda_{+}^{\alpha-4}+C_{-} \lambda_{-}^{\alpha-4}\right)}{\left(\Gamma\left(1-\frac{\alpha}{2}\right)\left(C_{+} \lambda_{+}^{\alpha-2}+C_{-} \lambda_{-}^{\alpha-2}\right)\right)^{2}} .
\end{aligned}
$$

The parameters $\lambda_{+}$and $\lambda_{-}$control the rate of decay on the positive and negative tails, respectively. If $\lambda_{+}>\lambda_{-}\left(\lambda_{+}<\lambda_{-}\right)$, then the distribution is skewed to the left (right). Moreover, if $\lambda_{+}=\lambda_{-}$, then it is symmetric. If we substitute

$$
C=C_{+}=C_{-}=2^{\frac{\alpha}{2}}\left(\Gamma\left(1-\frac{\alpha}{2}\right)\left(\lambda_{+}^{\alpha-2}+\lambda_{-}^{\alpha-2}\right)\right)^{-1}
$$

then $X \sim R D T S\left(\alpha, C, C, \lambda_{+}, \lambda_{-}, 0\right)$ has zero mean and unit variance. In this case, $X$ is called the standard RDTS distribution and denoted by $X \sim \operatorname{stdRDTS}\left(\alpha, \lambda_{+}, \lambda_{-}\right)$. Moreover, the log-Laplace transform of $X$ is denoted by $L_{R D T S}\left(x ; \alpha, \lambda_{+}, \lambda_{-}\right)$. By Proposition 3.4, the function $L_{R D T S}\left(x ; \alpha, \lambda_{+}, \lambda_{-}\right)$is finite for all $x \in \mathbb{R}$, and we have

$$
L_{R D T S}\left(x ; \alpha, \lambda_{+}, \lambda_{-}\right)=C G\left(x ; \alpha, \lambda_{+}\right)+C G\left(-x ; \alpha, \lambda_{-}\right) .
$$

Since the RDTS distribution is infinitely divisible, we can generate a Lévy process called the RDTS process.

Definition 3.6. A Lévy process $X=\left(X_{t}\right)_{t \geq 0}$ is said to be a RDTS process with param- 
eters $\left(\alpha, C_{+}, C_{-}, \lambda_{+}, \lambda_{-}, m\right)$ if $X_{1} \sim \operatorname{RDTS}\left(\alpha, C_{+}, C_{-}, \lambda_{+}, \lambda_{-}, m\right)$.

The parameter $\alpha$ determines the path behavior; that is, the RDTS process has finite variation if $\alpha<1$ and infinite variation if $\alpha>1$. The following proposition (which we prove in Appendix A) will be used for determining the equivalent martingale measure.

Proposition 3.7. Suppose $\left(X_{t}\right)_{t \in[0, T]}$ is the RDTS process with parameters $\left(\alpha, C_{+}, C_{-}\right.$, $\left.\lambda_{+}, \lambda_{-}, m\right)$ under $\mathbb{P}$, and the RDTS process with parameters $\left(\tilde{\alpha}, \tilde{C}_{+}, \tilde{C}_{-}, \tilde{\lambda}_{+}, \tilde{\lambda}_{-}, \tilde{m}\right)$ under $\mathbb{Q}$. Then $\left.\mathbb{P}\right|_{\mathcal{F}_{t}}$ and $\left.\mathbb{Q}\right|_{\mathcal{F}_{t}}$ are equivalent for all $t>0$ if and only if $\alpha=\tilde{\alpha}, C_{+}=\tilde{C}_{+}$, $C_{-}=\tilde{C}_{-}$, and

$$
\tilde{m}-m=2^{-\frac{\alpha+1}{2}} \Gamma\left(\frac{1-\alpha}{2}\right)\left(C_{+}\left(\tilde{\lambda}_{+}^{\alpha-1}-\lambda_{+}^{\alpha-1}\right)-C_{-}\left(\tilde{\lambda}_{-}^{\alpha-1}-\lambda_{-}^{\alpha-1}\right)\right)
$$

When $\mathbb{P}$ and $\mathbb{Q}$ are equivalent, the Radon-Nikodym derivative is $\left.\frac{d \mathbb{Q}}{d \mathbb{P}}\right|_{\mathcal{F}_{t}}=e^{U_{t}}$ where $\left(U_{t}, \mathbb{P}\right)$ is a Lévy process with Lévy triplet $\left(\sigma_{U}^{2}, \nu_{U}, \gamma_{U}\right)$ given by

$$
\sigma_{U}^{2}=0, \nu_{U}=\nu \circ \psi^{-1}, \gamma_{U}=-\int_{-\infty}^{\infty}\left(e^{y}-1-y 1_{|y| \leq 1}\right)\left(\nu \circ \psi^{-1}\right)(d y)
$$

where $\psi(x)=\frac{x^{2}}{2}\left(\lambda_{+}-\tilde{\lambda}_{+}\right) 1_{x>0}+\frac{x^{2}}{2}\left(\lambda_{-}-\tilde{\lambda}_{-}\right) 1_{x<0}$.

Applying Proposition 3.7 to RDTS distributed random variables, we can obtain the following corollary.

Corollary 3.8. (a) Let $X \sim \operatorname{RDTS}\left(\alpha, C_{+}, C_{-}, \lambda_{+}, \lambda_{-}, m\right)$ under a measure $\mathbf{P}$, and $X \sim \operatorname{RDTS}\left(\tilde{\alpha}, \tilde{C}_{+}, \tilde{C}_{-}, \tilde{\lambda}_{+}, \tilde{\lambda}_{-}, \tilde{m}\right)$ under a measure $\mathbf{Q}$. Then $\mathbf{P}$ and $\mathbf{Q}$ are equivalent if and only if $\alpha=\tilde{\alpha}, C_{+}=\tilde{C}_{+}, C_{-}=\tilde{C}_{-}$, and

$$
\tilde{m}-m=2^{-\frac{\alpha+1}{2}} \Gamma\left(\frac{1-\alpha}{2}\right)\left(C_{+}\left(\tilde{\lambda}_{+}^{\alpha-1}-\lambda_{+}^{\alpha-1}\right)-C_{-}\left(\tilde{\lambda}_{-}^{\alpha-1}-\lambda_{-}^{\alpha-1}\right)\right) .
$$

(b) Let $X \sim \operatorname{stdRDTS}\left(\alpha, \lambda_{+}, \lambda_{-}\right)$under a measure $\mathbf{P}$, and $(X+k) \sim \operatorname{stdRDTS}\left(\tilde{\alpha}, \tilde{\lambda}_{+}\right.$, $\tilde{\lambda}_{-}$) under a measure $\mathbf{Q}$ for a constant $k \in \mathbb{R}$. Then $\mathbf{P}$ and $\mathbf{Q}$ are equivalent if and only 


$$
\left\{\begin{array}{l}
\alpha=\tilde{\alpha} \\
\lambda_{+}^{\alpha-2}+\lambda_{-}^{\alpha-2}=\tilde{\lambda}_{+}^{\alpha-2}+\tilde{\lambda}_{-}^{\alpha-2} \\
k=\frac{\Gamma\left(\frac{1-\alpha}{2}\right)\left(\lambda_{+}^{\alpha-1}-\lambda_{-}^{\alpha-1}-\tilde{\lambda}_{+}^{\alpha-1}+\tilde{\lambda}_{-}^{\alpha-1}\right)}{\sqrt{2} \Gamma\left(1-\frac{\alpha}{2}\right)\left(\tilde{\lambda}_{+}^{\alpha-2}+\tilde{\lambda}_{-}^{\alpha-2}\right)} .
\end{array}\right.
$$

\subsection{Simulation of the RDTS distribution}

The RDTS distribution is included in the class of TID distributions. The general method of generating TID distributed random numbers can be found in Bianchi et al. (2008) and we summarize it below.

Consider $\alpha \in(0,2) \backslash\{1\}, C>0$, and $\lambda_{+}, \lambda_{-}>0$. Let $\left\{v_{j}\right\}$ be an i.i.d. sequence of random variables in $\left\{\lambda_{+}, \lambda_{-}\right\}$with $P\left(v_{j}=\lambda_{+}\right)=P\left(v_{j}=-\lambda_{-}\right)=1 / 2$. Let $\left\{u_{j}\right\}$ be an i.i.d. sequence of uniform random variables on $(0,1)$ and let $\left\{e_{j}\right\}$ and $\left\{e_{j}^{\prime}\right\}$ be i.i.d. sequences of exponential random variables with parameters 1 and $1 / 2$, respectively. Furthermore, we assume that $\left\{v_{j}\right\},\left\{u_{j}\right\},\left\{e_{j}\right\}$, and $\left\{e_{j}^{\prime}\right\}$ are independent. We consider $\gamma_{j}=e_{1}^{\prime}+\ldots+e_{j}^{\prime}$.

Using Theorems 4.2 and 4.3 of Bianchi et al. (2008), we can obtain the following theorem.

Theorem 3.9. Suppose that all the above assumptions are fulfilled. If $\alpha \in(0,2) \backslash\{1\}$, the series

$$
X=\sum_{j=1}^{\infty}\left(\left(\frac{\alpha \gamma_{j}}{C}\right)^{-1 / \alpha} \wedge \sqrt{2} e_{j}^{1 / 2} u_{j}^{1 / \alpha}\left|v_{j}\right|^{-1}\right) \frac{v_{j}}{\left|v_{j}\right|}-\frac{C \Gamma\left(\frac{1-\alpha}{2}\right)}{2^{\frac{\alpha+1}{2}}}\left(\lambda_{+}^{\alpha-1}-\lambda_{-}^{\alpha-1}\right)
$$

converges a.s. Furthermore, we have that $X \sim \operatorname{RDTS}\left(\alpha, C, C, \lambda_{+}, \lambda_{-}, 0\right)$.

\subsection{Tail properties}

Let's look at the probability tails of the CTS and RDTS distributions. Although the exact asymptotic behavior of its tails is difficult to obtain unlike those of the stable 
distribution, it is possible to calculate the upper and lower bounds.

Proposition 3.10. If $X \sim \operatorname{CTS}\left(\alpha, C_{+}, C_{-}, \lambda_{+}, \lambda_{-}, m\right)$, then the following inequality is fulfilled

$$
k \frac{e^{-2 \bar{\lambda} y}}{\bar{\lambda} y^{\alpha+1}} \leq \mathbb{P}(|X-m| \geq y) \leq \frac{K}{y^{2}}
$$

as $y \rightarrow \infty$, where $k$ and $K$ do not depend on $y$ and $\bar{\lambda}=\min \left(\lambda_{+}, \lambda_{-}\right)$.

Proposition 3.11. If $X \sim \operatorname{RDTS}\left(\alpha, C_{+}, C_{-}, \lambda_{+}, \lambda_{-}, m\right)$, then the following inequality is fulfilled

$$
k \frac{e^{-2 \bar{\lambda}^{2} y^{2}}}{\bar{\lambda}^{2} y^{\alpha+2}} \leq \mathbb{P}(|X-m| \geq y) \leq \frac{K}{y^{2}}
$$

as $y \rightarrow \infty$, where $k$ and $K$ do not depend on $y$ and $\bar{\lambda}=\min \left(\lambda_{+}, \lambda_{-}\right)$.

\section{GARCH model with infinitely divisible distributed in- novations}

Our objective in this section is twofold. First, we review the infinitely divisible GARCH (ID-GARCH) model and the CTS-GARCH model which is a subclass of the ID-GARCH model. Second, we construct a new subclass of the ID-GARCH model with standard RDTS distributed innovation. Some details and proofs for the ID-GARCH model and CTS-GARCH model can be found in Kim et al. (2008a).

The ID-GARCH stock price model is defined over a filtered probability space $(\Omega, \mathfrak{F}$, $\left.\left(\mathfrak{F}_{t}\right)_{t \in \mathbb{N}}, \mathbb{P}\right)$ which is constructed as follows. Consider a sequence $\left(\varepsilon_{t}\right)_{t \in \mathbb{N}}$ of i.i.d. real random variables on a sequence of probability spaces $\left(\Omega_{t}, \mathbf{P}_{t}\right)_{t \in \mathbb{N}}$, such that $\varepsilon_{t}$ is an ID distributed random variable with zero mean and unit variance on $\left(\Omega_{t}, \mathbf{P}_{t}\right)$, and assume that $E\left[e^{x \varepsilon_{t}}\right]<\infty$ where $x \in I$ for some real interval $I$ containing zero. Now we define $\Omega:=\prod_{t \in \mathbb{N}} \Omega_{t}, \mathfrak{F}_{t}:=\otimes_{k=1}^{t} \sigma\left(\varepsilon_{k}\right) \otimes \mathfrak{F}_{0} \otimes \mathfrak{F}_{0} \cdots, \mathfrak{F}:=\sigma\left(\cup_{t \in \mathbb{N}} \mathfrak{F}_{t}\right)$, and $\mathbb{P}:=\otimes_{t \in \mathbb{N}} \mathbf{P}_{t}$, where $\mathfrak{F}_{0}=\{\emptyset, \Omega\}$ and $\sigma\left(\varepsilon_{k}\right)$ means the $\sigma$-algebra generated by $\varepsilon_{k}$ on $\Omega_{k}$. 
We first propose the following stock price dynamic:

$$
\log \left(\frac{S_{t}}{S_{t-1}}\right)=r_{t}-d_{t}+\lambda_{t} \sigma_{t}-L\left(\sigma_{t}\right)+\sigma_{t} \varepsilon_{t}, \quad t \in \mathbb{N},
$$

where $S_{t}$ is the stock price at time $t, r_{t}$, and $d_{t}$ denote the risk-free and dividend rate for the period $[t-1, t]$, respectively, and $\lambda_{t}$ is a $\mathfrak{F}_{t-1}$ measurable random variable. $S_{0}$ is the currently observed price. The function $L(x)$ is the log-Laplace-transform of $\varepsilon_{t}$, i.e, $L(x)=\log \left(E\left[e^{x \varepsilon t}\right]\right)$. If $L(x)$ is defined on the whole real line, then the one-period ahead conditional variance $\sigma_{t}^{2}$ follows a $\operatorname{GARCH}(1,1)$ process, i.e,

$$
\sigma_{t}^{2}=\left(\alpha_{0}+\alpha_{1} \sigma_{t-1}^{2} \varepsilon_{t-1}^{2}+\beta_{1} \sigma_{t-1}^{2}\right), \quad t \in \mathbb{N}, \quad \varepsilon_{0}=0 .
$$

where $\alpha_{0}, \alpha_{1}$, and $\beta_{1}$ are non-negative, $\alpha_{1}+\beta_{1}<1$, and $\alpha_{0}>0$. If $L(x)$ is defined only on a closed interval $[-a, b]$ with $a, b>0$, then $\sigma_{t}^{2}$ follows a $\operatorname{GARCH}(1,1)$ process with a restriction $0<\sigma_{t} \leq b$, i.e,

$$
\sigma_{t}^{2}=\left(\alpha_{0}+\alpha_{1} \sigma_{t-1}^{2} \varepsilon_{t-1}^{2}+\beta_{1} \sigma_{t-1}^{2}\right) \wedge \rho, \quad t \in \mathbb{N}, \quad \varepsilon_{0}=0
$$

where $0<\rho \leq b^{2}$. Clearly, the process $\left(\sigma_{t}\right)_{t \in \mathbb{N}}$ is predictable. In "the normal-GARCH model" introduced by Duan (1995), for example, the Laplace transform of $\varepsilon_{t}$ is defined for every real number and hence $\sigma_{t}^{2}$ follows (4.2).

\subsection{CTS-GARCH Model}

Consider the ID-GARCH model with the sequence $\left(\varepsilon_{t}\right)_{t \in \mathbb{N}}$ of i.i.d. random variables with $\varepsilon_{t} \sim \operatorname{stdCTS}\left(\alpha, \lambda_{+}, \lambda_{-}\right)$for all $t \in \mathbb{N}$. This ID-GARCH model has been introduced by Kim et al. (2008a) under the name CTS-GARCH model. Since $E\left[e^{x \varepsilon_{t}}\right]<\infty$ if $x \in$ $\left[-\lambda_{-}, \lambda_{+}\right], \rho$ has to be in the interval $\left(0, \lambda_{+}{ }^{2}\right]$, and $\sigma_{t}$ follows equation (4.3).

By Corollary 2.3 (b), we can prove the following proposition.

Proposition 4.1. Consider the CTS-GARCH model. Let $T \in \mathbb{N}$ be a time horizon, and 
t a natural number such that $t \leq T$. Suppose $\tilde{\lambda}_{+}(t)$ and $\tilde{\lambda}_{-}(t)$ satisfy the following conditions:

$$
\left(\begin{array}{l}
\tilde{\lambda}_{+}(t)^{2} \geq \rho \\
\tilde{\lambda}_{+}(t)^{\alpha-2}+\tilde{\lambda}_{-}(t)^{\alpha-2}=\lambda_{+}{ }^{\alpha-2}+\lambda_{-}{ }^{\alpha-2} \\
\frac{\lambda_{+}{ }^{\alpha-1}-\lambda_{-}{ }^{\alpha-1}-\tilde{\lambda}_{+}(t)^{\alpha-1}+\tilde{\lambda}_{-}(t)^{\alpha-1}}{(1-\alpha)\left(\lambda_{+}{ }^{\alpha-2}+\lambda_{-}{ }^{\alpha-2}\right)} \\
\quad=\lambda_{t}+\frac{1}{\sigma_{t}}\left(L_{C T S}\left(\sigma_{t} ; \alpha, \tilde{\lambda}_{+}(t), \tilde{\lambda}_{-}(t)\right)-L_{C T S}\left(\sigma_{t} ; \alpha, \lambda_{+}, \lambda_{-}\right)\right)
\end{array}\right.
$$

Then there is a measure $\mathbf{Q}_{t}$ equivalent to $\mathbf{P}_{t}$ such that $\varepsilon_{t}+k_{t} \sim \operatorname{stdCTS}\left(\alpha, \tilde{\lambda}_{+}(t), \tilde{\lambda}_{-}(t)\right)$ on the measure $\mathbf{Q}_{t}$ where $k_{t}$ is the $\mathcal{F}_{t-1}$ measurable random variable given by

$$
k_{t}=\lambda_{t}+\frac{1}{\sigma_{t}}\left(L_{C T S}\left(\sigma_{t} ; \alpha, \tilde{\lambda}_{+}(t), \tilde{\lambda}_{-}(t)\right)-L_{C T S}\left(\sigma_{t} ; \alpha, \lambda_{+}, \lambda_{-}\right)\right)
$$

Suppose $\tilde{\lambda}_{+}(t)$ and $\tilde{\lambda}_{-}(t)$ satisfy the condition (4.4) in each time $t \leq T$. We have the stock price dynamic

$$
\log \left(\frac{S_{t}}{S_{t-1}}\right)=r_{t}-d_{t}-L_{C T S}\left(\sigma_{t} ; \alpha, \tilde{\lambda}_{+}(t), \tilde{\lambda}_{-}(t)\right)+\sigma_{t}\left(\varepsilon_{t}+k_{t}\right)
$$

where $k_{t}$ is given by equation (4.5). By Proposition 4.1, there is a measure $\mathbf{Q}_{t}$ equivalent to $\mathbf{P}_{t}$ such that $\varepsilon_{t}+k_{t} \sim \operatorname{stdCTS}\left(\alpha, \tilde{\lambda}_{+}(t), \tilde{\lambda}_{-}(t)\right)$ on the measure $\mathbf{Q}_{t}$, and hence we obtain a risk-neutral stock price dynamic

$$
\left\{\begin{array}{l}
\log \left(\frac{S_{t}}{S_{t-1}}\right)=r_{t}-d_{t}-L_{C T S}\left(\sigma_{t} ; \alpha, \tilde{\lambda}_{+}(t), \tilde{\lambda}_{-}(t)\right)+\sigma_{t} \xi_{t} \\
\xi_{t} \sim \operatorname{stdCTS}\left(\alpha, \tilde{\lambda}_{+}(t), \tilde{\lambda}_{-}(t)\right)
\end{array}, t \leq T\right.
$$

having the following variance process

$$
\sigma_{t}^{2}=\left(\alpha_{0}+\alpha_{1} \sigma_{t-1}^{2}\left(\xi_{t-1}-k_{t-1}\right)^{2}+\beta_{1} \sigma_{t-1}^{2}\right) \wedge \rho .
$$

The risk-neutral stock price dynamic is called the CTS-GARCH option pricing model. 
Under the CTS-GARCH option pricing model, the stock price $S_{t}$ at time $t>0$ is given by

$$
S_{t}=S_{0} \exp \left(\sum_{j=1}^{t}\left(r_{j}-d_{j}-L_{C T S}\left(\sigma_{j} ; \alpha, \tilde{\lambda}_{+}(j), \tilde{\lambda}_{-}(j)\right)+\sigma_{j} \xi_{j}\right)\right)
$$

\subsection{RDTS-GARCH Model}

Consider the ID-GARCH model with the sequence $\left(\varepsilon_{t}\right)_{t \in \mathbb{N}}$ of i.i.d. random variables with $\varepsilon_{t} \sim \operatorname{stdRDTS}\left(\alpha, \lambda_{+}, \lambda_{-}\right)$for all $t \in \mathbb{N}$. We will call the ID-GARCH model the RDTS-GARCH model. Since $E\left[e^{x \varepsilon_{t}}\right]<\infty$ for all real number $x$, the variance process is not artificially restricted; that is, $\sigma_{t}$ follows (4.2).

By Corollary 3.8 (b), we can prove the following proposition.

Proposition 4.2. Consider the RDTS-GARCH model. Let $T \in \mathbb{N}$ be a time horizon, and $t$ a natural number such that $t \leq T$. Suppose $\tilde{\lambda}_{+}(t)$ and $\tilde{\lambda}_{-}(t)$ satisfy the following conditions:

$$
\left(\begin{array}{l}
\tilde{\lambda}_{+}(t)^{\alpha-2}+\tilde{\lambda}_{-}(t)^{\alpha-2}=\lambda_{+}^{\alpha-2}+\lambda_{-}^{\alpha-2} \\
\frac{\Gamma\left(\frac{1-\alpha}{2}\right)\left(\lambda_{+}^{\alpha-1}-\lambda_{-}^{\alpha-1}-\tilde{\lambda}_{+}(t)^{\alpha-1}+\tilde{\lambda}_{-}(t)^{\alpha-1}\right)}{\sqrt{2} \Gamma\left(1-\frac{\alpha}{2}\right)\left(\lambda_{+}^{\alpha-2}+\lambda_{-}^{\alpha-2}\right)} \\
\quad=\lambda_{t}+\frac{1}{\sigma_{t}}\left(L_{R D T S}\left(\sigma_{t} ; \alpha, \tilde{\lambda}_{+}(t), \tilde{\lambda}_{-}(t)\right)-L_{R D T S}\left(\sigma_{t} ; \alpha, \lambda_{+}, \lambda_{-}\right)\right)
\end{array}\right.
$$

Then there is a measure $\mathbf{Q}_{t}$ equivalent to $\mathbf{P}_{t}$ such that $\varepsilon_{t}+k_{t} \sim \operatorname{stdRDTS}\left(\alpha, \tilde{\lambda}_{+}(t), \tilde{\lambda}_{-}(t)\right)$ on the measure $\mathbf{Q}_{t}$ where $k_{t}$ is the $\mathcal{F}_{t-1}$ measurable random variable given by

$$
k_{t}=\lambda_{t}+\frac{1}{\sigma_{t}}\left(L_{R D T S}\left(\sigma_{t} ; \alpha, \tilde{\lambda}_{+}(t), \tilde{\lambda}_{-}(t)\right)-L_{R D T S}\left(\sigma_{t} ; \alpha, \lambda_{+}, \lambda_{-}\right)\right) .
$$

Suppose $\tilde{\lambda}_{+}(t)$ and $\tilde{\lambda}_{-}(t)$ satisfy condition (4.8) in each time $t \leq T$. We would then 
have the stock price dynamic

$$
\begin{aligned}
\log \left(\frac{S_{t}}{S_{t-1}}\right) & =r_{t}-d_{t}+\lambda_{t} \sigma_{t}-L_{R D T S}\left(\sigma_{t} ; \alpha, \lambda_{+}, \lambda_{-}\right)+\sigma_{t} \varepsilon_{t} \\
& =r_{t}-d_{t}-L_{R D T S}\left(\sigma_{t} ; \alpha, \tilde{\lambda}_{+}(t), \tilde{\lambda}_{-}(t)\right)+\sigma_{t}\left(\varepsilon_{t}+k_{t}\right)
\end{aligned}
$$

where $k_{t}$ is given by equation (4.9). By Proposition 4.2, there is a measure $\mathbf{Q}_{t}$ equivalent to $\mathbf{P}_{t}$ such that $\varepsilon_{t}+k_{t} \sim \operatorname{stdRDTS}\left(\alpha, \tilde{\lambda}_{+}(t), \tilde{\lambda}_{-}(t)\right)$ on the measure $\mathbf{Q}_{t}$, and hence

$$
\log \left(\frac{S_{t}}{S_{t-1}}\right)=r_{t}-d_{t}-L_{R D T S}\left(\sigma_{t} ; \alpha, \tilde{\lambda}_{+}(t), \tilde{\lambda}_{-}(t)\right)+\sigma_{t} \xi_{t}
$$

where $\xi_{t} \sim \operatorname{stdRDTS}\left(\alpha, \tilde{\lambda}_{+}(t), \tilde{\lambda}_{-}(t)\right)$. Since $\lambda_{t} \sigma_{t}$ disappears in the dynamic on $\mathbf{Q}_{t}, \lambda_{t}$ can be interpreted as the market price of risk. Consequently, we deduce the following risk-neutral stock price dynamic from Proposition 4.2

$$
\left\{\begin{array}{l}
\log \left(\frac{S_{t}}{S_{t-1}}\right)=r_{t}-d_{t}-L_{R D T S}\left(\sigma_{t} ; \alpha, \tilde{\lambda}_{+}(t), \tilde{\lambda}_{-}(t)\right)+\sigma_{t} \xi_{t} \quad, t \leq T \\
\xi_{t} \sim \operatorname{stdRDTS}\left(\alpha, \tilde{\lambda}_{+}(t), \tilde{\lambda}_{-}(t)\right)
\end{array}\right.
$$

having the following variance process

$$
\sigma_{t}^{2}=\left(\alpha_{0}+\alpha_{1} \sigma_{t-1}^{2}\left(\xi_{t-1}-k_{t-1}\right)^{2}+\beta_{1} \sigma_{t-1}^{2}\right)
$$

The risk-neutral stock price dynamic is called the RDTS-GARCH option pricing model. Under the RDTS-GARCH option pricing model, the stock price $S_{t}$ at time $t>0$ is given by

$$
S_{t}=S_{0} \exp \left(\sum_{j=1}^{t}\left(r_{j}-d_{j}-L_{R D T S}\left(\sigma_{j} ; \alpha, \tilde{\lambda}_{+}(j), \tilde{\lambda}_{-}(j)\right)+\sigma_{j} \xi_{j}\right)\right)
$$

\subsection{Simulation of the risk-neutral stock price processes}

Assume that the GARCH parameters $\left(\alpha_{0}, \alpha_{1}\right.$, and $\left.\beta_{1}\right)$, the standard CTS and standard RDTS parameters $\left(\alpha, \lambda_{+}\right.$, and $\left.\lambda_{-}\right)$, the constant market price of risk $\lambda_{t}=\lambda$, and the 
conditional variance $\sigma_{t_{0}}^{2}$ of the initial time $t_{0}$ are estimated from historical data. Then we can generate the risk-neutral process for the CTS-GARCH option pricing model by the following algorithm:

1. Initialize $t:=t_{0}$.

2. Find the parameters $\tilde{\lambda}_{+}(t)$ and $\tilde{\lambda}_{-}(t)$ satisfying condition (4.4).

3. Generate random number $\xi_{t} \sim \operatorname{stdCTS}\left(\alpha, \tilde{\lambda}_{+}(t), \tilde{\lambda}_{-}(t)\right)$ using Theorem 2.4.

4. Let $\log \left(\frac{S_{t}}{S_{t-1}}\right)$ be equal to equation (4.6).

5. Let $k_{t}$ be equal to equation (4.5).

6. Set $t=t+1$ and then substitute

$$
\sigma_{t}^{2}=\left(\alpha_{0}+\alpha_{1} \sigma_{t-1}^{2}\left(\xi_{t-1}-k_{t-1}\right)^{2}+\beta_{1} \sigma_{t-1}^{2}\right) \wedge \rho
$$

7. Repeat 2 through 6 until $t>T$.

We can generate the risk-neutral process for the RDTS-GARCH option pricing model by modifying the above algorithm as follows:

$2^{\prime}$. Find the parameters $\tilde{\lambda}_{+}(t)$ and $\tilde{\lambda}_{-}(t)$ satisfying condition (4.8).

$3^{\prime}$. Generate random number $\xi_{t} \sim \operatorname{stdRDTS}\left(\alpha, \tilde{\lambda}_{+}(t), \tilde{\lambda}_{-}(t)\right)$ using Theorem 3.9.

$4^{\prime}$. Let $\log \left(\frac{S_{t}}{S_{t-1}}\right)$ be equal to equation (4.10).

$5^{\prime}$. Let $k_{t}$ be equal to equation (4.9).

$6^{\prime}$. Set $t=t+1$ and then substitute

$$
\sigma_{t}^{2}=\alpha_{0}+\alpha_{1} \sigma_{t-1}^{2}\left(\xi_{t-1}-k_{t-1}\right)^{2}+\beta_{1} \sigma_{t-1}^{2}
$$

\section{Market parameter estimation}

In this section, we report the maximum likelihood estimation (MLE) of the normalGARCH, CTS-GARCH, and RDTS-GARCH models using data obtained from Option Metrics's IvyDB in the Wharton Research Data Services. In our empirical study, we use historical prices of the Dow Jones Industrial Average (DJIA) and 29 of its 30-component 
stocks $^{2}$ as of October 2008. First, we consider the time series of the stock prices for the DJIA component companies from October 1, 1997 to December 31, 2006. Then in order to analyze the model performance during that time and to evaluate DJX index options, we consider also the time series of the DJIA index in the time window from January 2, 1996 to June 6,2007 . The analysis of the 29 stocks is totally independent of the analysis of the DJIA and DJX. That is, we study the model performances on stocks, then on the DJIA index together with the corresponding option prices. Since the index composition changes periodically, we prefer to perform the analysis on the current DJIA component stocks. For the daily risk-free rate, we select the appropriate zero-coupon rate obtained from the Ivy DB.

To simplify the estimation, we impose a constant market price of risk $\lambda$. We use the total returns data by Ivy DB to estimate the market parameters with the MLE. The total returns are obtained by adjusting prices of indexes and stocks for all applicable splits and dividend distributions. For this reason, we modify the stock price dynamic as follows

$$
\log \left(\frac{\hat{S}_{t}}{\hat{S}_{t-1}}\right)=r_{t}+\lambda_{t} \sigma_{t}-L\left(\sigma_{t}\right)+\sigma_{t} \varepsilon_{t}, t \in \mathbb{N}
$$

where $\hat{S}_{t}$ is the adjusted-closing prices.

Our estimation procedure is as follows. First, we estimate the parameters $\alpha_{0}, \alpha_{1}$, $\beta_{1}$, and the constant market price of risk $\lambda$ from the normal-GARCH model. Second, we fix $\alpha_{0}, \alpha_{1}, \beta_{1}$, and $\lambda$ as parameters estimated in the first step and then estimate $\alpha, \lambda_{+}$, and $\lambda_{-}$from the CTS-GARCH and RDTS-GARCH models under the assumption of $\sigma_{0}^{2}=\alpha_{0} /\left(1-\alpha_{1}-\beta_{1}\right)$. For the CTS-GARCH model, we set $\rho=\max \left\{\sigma_{t}^{2}\right.$ : $t$ is the observed date $\}$. We report the estimated GARCH parameters in Table 1, and the parameters for the two standard tempered stable distributions in Table 2 for the DJIA index and 29 component companies.

For the assessment of the goodness-of-fit, we utilize the Kolmogrov-Smirnov (KS) test. We also calculate the Anderson-Darling (AD) statistic to better evaluate the tail fit. We define the null hypotheses as follows: 
$H_{0}$ (Normal-GARCH): The residuals follow the standard normal distribution.

$H_{0}(\mathrm{CTS}-\mathrm{GARCH})$ : The residuals follow the standard CTS distribution.

$H_{0}($ RDTS-GARCH): The residuals follow the standard RDTS distribution.

Table 3 provides the KS statistic and its $p$-values. The $p$-values of the KS statistic are calculated using the calculator designed by Marsaglia et al. (2003). Based on the results reported in the table, we conclude that

1. $H_{0}($ Normal-GARCH $)$ is rejected at the $5 \%$ significance level for 22 of the 29 stocks.

2. $H_{0}(\mathrm{CTS}-\mathrm{GARCH})$ is rejected at the $5 \%$ significance level for one stock, DuPont.

3. $H_{0}(\mathrm{RDTS}-\mathrm{GARCH})$ is rejected at the $5 \%$ significance level for one stock, DuPont.

4. AD statistic for both CTS-GARCH and RDTS-GARCH are significantly smaller than the AD statistic of the normal-GARCH model.

Furthermore, in order to analyze the model performance during the time, we report the MLE estimate of the normal-GARCH, CTS-GARCH, and RDTS-GARCH models for the DJIA, by considering any Wednesday between January 4, 2006 to June 6, 2007. We consider 75 different time series with daily observations starting from January 2 , 1996 and ending on any Wednesday in the time window considered above. This estimations will be also used in the next section for the purpose of option valuation. We report in Table 4 and Figure 1 the normal-GARCH parameters, and in Table 5 and in Figure 2 the market parameters of the innovation processes for the CTS-GARCH and RDTS-GARCH models. In Table 6 and Figure 3, we show the KS, the AD, and the $\chi^{2}$ statistic with the relative $p$-value. The empirical study shows that the two non-normal GARCH models largely improve the classical normal-GARCH model. Furthermore, Figure 2 shows that the estimated parameters of the CTS and RDTS innovations do not present large deviations in a time window of more than one year, and, in particular, the RDTS model parameters seem to be more stable. 


\section{Option prices with GARCH models}

In this part of the empirical analysis, we evaluate option prices written on the DJIA (DJX) with different strike prices and maturities. Now, we want to study the effect on option prices when the underlying distribution is skewed and leptokurtic, and compare these models to the normal-GARCH model, which constitute a natural benchmark. European call data on 17 selected Wednesdays (one per month) between January 4, 2006 and May 9, 2007 are considered for a total of 2,670 option prices. Here, options with a time to maturity more than 250 days are discarded. Option prices and the risk-free rate, calculated from the U.S. Treasury yield curve, are provided by Ivy DB.

Market parameters estimated in the previous section are taken into account in this analysis in order to calculate option prices. We consider the market estimation based on the time series from January 2, 1996 to any corresponding Wednesday in which the European call option is quoted. That is, to price an option quoted on January 11, 2006, we consider the MLE estimated parameters from the time series from January 2, 1996 to January 11, 2006, together with the algorithms in Section 4.3. By repeating the same procedure, we price options for any selected Wednesday, until May 9, 2007.

The Monte Carlo procedure is based on algorithms in Section 4.3 with empirical martingale simulation. This simulation technique, introduced in Duan and Simonato (1998), is a simple way to reduce the variance of the simulated sample and to preserve the martingale property of the simulated risk-neutral process as well, which is in general lost with a crude Monte Carlo method. We point out that for each time step and for each simulated path, we have to solve a nonlinear system, as described in Section 4.3, to find risk-neutral parameters. That is, each random number may have different parameters, which does not occur in the normal case. For this reason, the running time ranges from 10 minutes for the normal case to 42 hours for the RDTS case to simulate 20,000 paths, by using Matlab R2007b on a Xeon Precision at 3.0 GHz with 3GB RAM. Anyway, if one can compute with a cluster, the running time is of minor concern, since the structure of the problem allows one to simulate paths separately. Furthermore, we have to also 
consider some memory allocation feature in working with an office personal computer such as a Xeon Precision at 3.0 GHz. This is the reason why we consider only 17 Wednesday, one per month, and not all 75 Wednesday as in the market estimation.

To measure the performance of the option pricing model, we consider four statistics (see Schoutens (2003)), described as follows. Let us consider a given market model and observed prices $C_{i}$ of call options with maturities $\tau_{i}$ and strikes $K_{i}, i \in\{1, \ldots, N\}$, where $N$ is the number of options on a given Wednesday. Let $\bar{C}_{i}$ be the mean of options prices $C_{i}$ and $\widehat{C}_{i}$ be the model price, then we evaluate

1. the average absolute error as a percentage of the mean price (denoted APE)

$$
\mathrm{APE}=\frac{1}{\bar{C}_{i}} \sum_{i=1}^{N} \frac{\left|C_{i}-\widehat{C}_{i}\right|}{N}
$$

2. the average absolute error (denoted AAE)

$$
\mathrm{AAE}=\sum_{i=1}^{N} \frac{\left|C_{i}-\widehat{C}_{i}\right|}{N},
$$

3. the root mean square error (denoted RMSE)

$$
\operatorname{RMSE}=\sqrt{\sum_{i=1}^{N} \frac{\left(C_{i}-\widehat{C}_{i}\right)^{2}}{N}},
$$

4. the average relative percentage error (denoted ARPE)

$$
\mathrm{ARPE}=\frac{1}{N} \sum_{i=1}^{N} \frac{\left|C_{i}-\widehat{C}_{i}\right|}{C_{i}}
$$

Table 7 reports the performance of different option pricing model: the normalGARCH performs worst than the two others models, as the CTS-GARCH and RDTSGARCH models have smaller pricing errors. 


\section{Conclusion}

In this paper, we introduce the RDTS distribution. It has statistical properties similar to the CTS distribution, even if the RDTS distribution has finite exponential moment of any order, while the CTS has only some finite exponential moment. Furthermore, we present a discrete time model for stock price log returns driven by a non-normal random variable, that is the RDTS-GARCH model, which allows fat tails, skewness, and volatility clustering. We compare this model to the classical normal-GARCH model and with the CTS-GARCH model, that was introduced by Kim et al. (2008a).

Discrete time markets with a continuous return distribution fail to be complete. Consequently, based on a similar argument as in the CTS case as per Kim et al. (2008a), the problem of the appropriate choice of the equivalent martingale for the discounted asset price process is solved considering the RDTS innovation assumption. A density transformation between ID random variables allows us to choice a suitable equivalent martingale measure. By the discrete time nature of this setting, the risk-neutral distribution is not always the same for the entire time window, but on each time step it is governed by different parameters. Unfortunately, this approach does not provide analytical solutions to price European options and hence numerical procedures have to be considered. For this reason, algorithms for simulating CTS and RDTS distributions are studied and used to obtain option prices. The use of non-normal GARCH models combined with Monte Carlo simulation methods allows one to obtain very promising results.

For the stocks, the index, and the option prices we analyzed and for the time period studied, the CTS-GARCH and RDTS-GARCH seem to be satisfactory in both market and option analysis, compared to the normal-GARCH model. Consequently, the CTSGARCH and RDTS-GARCH models explain both the asset price behavior and European option prices better than the normal-GARCH model. Thus, we can say that the skewness and fat-tail properties of the innovation are also important for pricing of European options. 


\section{A Appendix}

\section{A.1 Proof of Proposition 3.3}

Lemma A.1. If $\lambda>0$, then

$$
\int_{0}^{\infty} x^{n} \frac{e^{-\lambda^{2} x^{2} / 2}}{x^{\alpha+1}} d x=\frac{1}{2}\left(\frac{\lambda^{2}}{2}\right)^{\frac{\alpha-n}{2}} \Gamma\left(\frac{n-\alpha}{2}\right), \quad n \in \mathbb{N} .
$$

Proof. By the change of variables, we have

$$
\int_{0}^{\infty} x^{n} \frac{e^{-y x^{2}}}{x^{\alpha+1}} d x=\frac{y^{(\alpha-n) / 2}}{2} \Gamma\left(\frac{n-\alpha}{2}\right), \quad n \in \mathbb{N}, y>0 .
$$

If we substitute $y=\lambda^{2} / 2$ in (A.2), then we obtain the result.

Lemma A.2. Let $\alpha \in(0,2), \alpha \neq 1$. Then we have

$$
\begin{aligned}
\sum_{n=0}^{\infty} \frac{1}{n !} \Gamma\left(\frac{n-\alpha}{2}\right) x^{n} & =\Gamma\left(-\frac{\alpha}{2}\right) M\left(-\frac{\alpha}{2}, \frac{1}{2} ;\left(\frac{x}{2}\right)^{2}\right) \\
& +x \Gamma\left(\frac{1-\alpha}{2}\right) M\left(\frac{1-\alpha}{2}, \frac{3}{2} ;\left(\frac{x}{2}\right)^{2}\right) .
\end{aligned}
$$

where $M$ is the confluent hypergeometric function Andrews (1998).

Proof. We have

$$
\begin{aligned}
\sum_{n=0}^{\infty} \frac{1}{n !} \Gamma\left(\frac{n-\alpha}{2}\right) x^{n} & =\sum_{n=0}^{\infty} \frac{1}{(2 n) !} \Gamma\left(n-\frac{\alpha}{2}\right) x^{2 n} \\
& +\sum_{n=0}^{\infty} \frac{1}{(2 n+1) !} \Gamma\left(n+\frac{1-\alpha}{2}\right) x^{2 n+1}
\end{aligned}
$$

By the facts that

$$
(2 n) !=n ! 2^{2 n}\left(\frac{1}{2}\right)_{n}, \quad(2 n+1) !=n ! 2^{2 n}\left(\frac{3}{2}\right)_{n},
$$


and

$$
\Gamma(n+y)=(y)_{n} \Gamma(y),
$$

we obtain

$$
\begin{aligned}
& \sum_{n=0}^{\infty} \frac{1}{n !} \Gamma\left(\frac{n-\alpha}{2}\right) x^{n} \\
& =\Gamma\left(-\frac{\alpha}{2}\right) \sum_{n=0}^{\infty} \frac{\left(-\frac{\alpha}{2}\right)_{n}}{n ! 2^{2 n}\left(\frac{1}{2}\right)_{n}} x^{2 n}+x \Gamma\left(\frac{1-\alpha}{2}\right) \sum_{n=0}^{\infty} \frac{\left(\frac{1-\alpha}{2}\right)_{n}}{n ! 2^{2 n}\left(\frac{3}{2}\right)_{n}} x^{2 n} \\
& =\Gamma\left(-\frac{\alpha}{2}\right) M\left(-\frac{\alpha}{2}, \frac{1}{2} ;\left(\frac{x}{2}\right)^{2}\right)+x \Gamma\left(\frac{1-\alpha}{2}\right) M\left(\frac{1-\alpha}{2}, \frac{3}{2} ;\left(\frac{x}{2}\right)^{2}\right)
\end{aligned}
$$

Proof of Proposition 3.3. We have

$$
\begin{aligned}
& \int_{-\infty}^{\infty}\left(e^{i u x}-1-i u x 1_{|x| \leq 1}\right) \nu(d x) \\
& =i u \int_{|x|>1} x \nu(d x)+\int_{0}^{\infty}\left(e^{i u x}-1-i u x\right) \nu(d x)+\int_{-\infty}^{0}\left(e^{i u x}-1-i u x\right) \nu(d x) \\
& =i u \int_{|x|>1}^{\infty} x \nu(d x) \\
& +C_{+} \sum_{n=2}^{\infty} \frac{1}{n !}(i u)^{n} \int_{0}^{\infty} x^{n} \frac{e^{-\lambda_{+}^{2} x^{2} / 2}}{x^{\alpha+1}} d x+C_{-} \sum_{n=2}^{\infty} \frac{1}{n !}(-i u)^{n} \int_{0}^{\infty} x^{n} \frac{e^{-\lambda_{-}^{2} x^{2} / 2}}{x^{\alpha+1}} d x
\end{aligned}
$$

By (A.1) and (A.3), we have

$$
\begin{aligned}
& \sum_{n=2}^{\infty} \frac{1}{n !}( \pm i u)^{n} \int_{0}^{\infty} x^{n} \frac{e^{-\lambda_{ \pm}^{2} x^{2} / 2}}{x^{\alpha+1}} d x \\
& =\sum_{n=0}^{\infty} \frac{1}{n !} \frac{1}{2}\left(\frac{\lambda_{ \pm}}{\sqrt{2}}\right)^{\alpha-n} \Gamma\left(\frac{n-\alpha}{2}\right)( \pm i u)^{n} \\
& \quad \mp i u \frac{1}{2}\left(\frac{\lambda_{ \pm}}{\sqrt{2}}\right)^{\alpha-1} \Gamma\left(\frac{1-\alpha}{2}\right)-\frac{1}{2}\left(\frac{\lambda_{ \pm}}{\sqrt{2}}\right)^{\alpha} \Gamma\left(\frac{-\alpha}{2}\right) \\
& =2^{-\frac{\alpha}{2}-1} \lambda_{ \pm}^{\alpha}\left[\Gamma\left(-\frac{\alpha}{2}\right)\left(M\left(-\frac{\alpha}{2}, \frac{1}{2} ; \frac{( \pm i u)^{2}}{2 \lambda_{ \pm}^{2}}\right)-1\right)\right. \\
& \left.+\frac{ \pm \sqrt{2} i u}{\lambda_{ \pm}} \Gamma\left(\frac{1-\alpha}{2}\right)\left(M\left(\frac{1-\alpha}{2}, \frac{3}{2} ; \frac{( \pm i u)^{2}}{2 \lambda_{ \pm}^{2}}\right)-1\right)\right]
\end{aligned}
$$


By Lévy-Khintchine formula and (3.1) in Definition 3.1, we obtain the characteristic function. Moreover, $\phi(u)$ can be extended via analytic continuation to the complex field $\mathbb{C}$.

\section{A.2 Proof of Proposition 3.7}

In this section, we review a general result of equivalence of measures presented by Sato (1999) and then apply it to the RDTS process.

Theorem A.3 (Sato (1999) Theorem 33.1 and 33.2.). Let $\left(X_{t}, \mathbb{P}\right)$ and $\left(X_{t}, \mathbb{Q}\right)$ be a Lévy processes on $\mathbb{R}$ with Lévy triplets $\left(\sigma^{2}, \nu, \gamma\right)$ and $\left(\tilde{\sigma}^{2}, \tilde{\nu}, \tilde{\gamma}\right)$ respectively. Then $\left.\mathbb{P}\right|_{\mathcal{F}_{t}}$ and $\left.\mathbb{Q}\right|_{\mathcal{F}_{t}}$ are equivalent for all $t>0$ if and only if the Lévy triplets satisfy

$$
\sigma^{2}=\tilde{\sigma}^{2}
$$

$$
\int_{-\infty}^{\infty}\left(e^{\psi(x) / 2}-1\right)^{2} \nu(d x)<\infty
$$

with the function $\psi(x)=\ln \left(\frac{\tilde{\nu}(d x)}{\nu(d x)}\right)$ and if $\sigma^{2}=0$ then

$$
\tilde{\gamma}-\gamma=\int_{|x| \leq 1} x(\tilde{\nu}-\nu)(d x)
$$

When $\mathbb{P}$ and $\mathbb{Q}$ are equivalent, the Radon-Nikodym derivative is

$$
\left.\frac{d \mathbb{Q}}{d \mathbb{P}}\right|_{\mathcal{F}_{t}}=e^{U_{t}}
$$

where $\left(U_{t}, \mathbf{P}\right)$ is a Lévy process in which the Lévy triplet $\left(\sigma_{U}^{2}, \nu_{U}, \gamma_{U}\right)$ of $\left(U_{t}\right)_{t \in[0, T]}$ is given by

$$
\sigma_{U}^{2}=\sigma^{2} \eta^{2}, \nu_{U}=\nu \circ \psi^{-1}, \gamma_{U}=-\frac{\sigma^{2} \eta^{2}}{2}-\int_{-\infty}^{\infty}\left(e^{y}-1-y 1_{|y| \leq 1}\right) \nu_{U}(d y)
$$


Here $\eta$ is such that

$$
\tilde{\gamma}-\gamma-\int_{|x| \leq 1} x(\tilde{\nu}-\nu)(d x)=\sigma^{2} \eta
$$

if $\sigma>0$ and zero if $\sigma=0$.

Since RDTS distributions are infinitely divisible, we can apply Theorem A.3 to obtain the change of measure.

Proof of Proposition 3.7. Let $(0, \nu, \gamma)$ and $(0, \tilde{\nu}, \tilde{\gamma})$ be Lévy triplets of $\left(X_{t}, \mathbb{P}\right)$ and $\left(X_{t}, \mathbb{Q}\right)$, respectively. Since the diffusion coefficients of RDTS processes are zero, (A.4) is satisfied. From the definition of Lévy measure $\tilde{\nu}$ and $\nu, \psi(x)$ in the condition (A.5) is equal to

$$
\begin{aligned}
\psi(x)= & \left(\ln \left(\frac{\tilde{C}_{+} x^{-\tilde{\alpha}}}{C_{+} x^{-\alpha}}\right)+\frac{x^{2}}{2}\left(\lambda_{+}-\tilde{\lambda}_{+}\right)\right) 1_{x>0} \\
& +\left(\ln \left(\frac{\tilde{C}_{-}|x|^{-\tilde{\alpha}}}{C_{-}|x|^{-\alpha}}\right)+\frac{x^{2}}{2}\left(\lambda_{-}-\tilde{\lambda}_{-}\right)\right) 1_{x<0} .
\end{aligned}
$$

Let $k(x)=\frac{\lambda_{+}^{2} x^{2}}{2} 1_{x>0}+\frac{\lambda_{-}^{2} x^{2}}{2} 1_{x<0}$ and $\tilde{k}(x)=\frac{\tilde{\lambda}_{+}^{2} x^{2}}{2} 1_{x>0}+\frac{\tilde{\lambda}_{-}^{2} x^{2}}{2} 1_{x<0}$. If $\alpha<\tilde{\alpha}$, then we have

$$
\lim _{x \rightarrow 0^{+}}\left(\frac{\sqrt{\tilde{C}_{+}} e^{-\tilde{k}(x) / 2}}{x^{(\tilde{\alpha}+1) / 2}}-\frac{\sqrt{C_{+}} e^{-k(x) / 2}}{x^{(\alpha+1) / 2}}\right)^{2} /\left(\frac{1}{x^{\tilde{\alpha}+1}}\right)=\tilde{C}_{+} .
$$

If $\alpha=\tilde{\alpha}$ but $C_{+} \neq \tilde{C}_{+}$, then we have

$$
\lim _{x \rightarrow 0^{+}}\left(\frac{\sqrt{\tilde{C}_{+}} e^{-\tilde{k}(x) / 2}}{x^{(\tilde{\alpha}+1) / 2}}-\frac{\sqrt{C_{+}} e^{-k(x) / 2}}{x^{(\alpha+1) / 2}}\right)^{2} /\left(\frac{1}{x^{\tilde{\alpha}+1}}\right)=\left(\sqrt{\tilde{C}_{+}}-\sqrt{C_{+}}\right)^{2} .
$$

Hence if $\alpha<\tilde{\alpha}$ or $\alpha=\tilde{\alpha}$ but $C_{+} \neq \tilde{C}_{+}$then

$$
\begin{aligned}
\int_{0}^{\infty}\left(e^{\psi(x) / 2}-1\right)^{2} \nu(d x) & =\int_{0}^{\infty}\left(\frac{\sqrt{\tilde{C}_{+}} e^{-\tilde{k}(x) / 2}}{x^{(\alpha+1) / 2}}-\frac{\sqrt{C_{+}} e^{-k(x) / 2}}{x^{(\alpha+1) / 2}}\right)^{2} d x \\
& \geq K_{+} \int_{0}^{1} \frac{1}{x^{\tilde{\alpha}+1}} d x=\infty
\end{aligned}
$$


for some $K_{+} \in \mathbb{R}$. Using similar arguments, we can prove that if $\alpha<\tilde{\alpha}$ or $\alpha=\tilde{\alpha}$ but $C_{-} \neq \tilde{C}_{-}$then

$$
\int_{-\infty}^{0}\left(e^{\psi(x) / 2}-1\right)^{2} \nu(d x)=\infty
$$

By (A.9) and (A.10),

$$
\int_{-\infty}^{\infty}\left(e^{\psi(x) / 2}-1\right)^{2} \nu(d x)=\infty
$$

Hence the condition (A.5) does not hold. Similarly, we can show that the condition (A.5) does not hold if $\alpha>\tilde{\alpha}$.

Suppose $\alpha=\tilde{\alpha}, C_{+}=\tilde{C}_{+}$and, $C_{-}=\tilde{C}_{-}$. Then we have

$$
\psi(x)=\frac{x^{2}}{2}\left(\lambda_{+}^{2}-\tilde{\lambda}_{+}^{2}\right) 1_{x>0}+\frac{x^{2}}{2}\left(\lambda_{-}^{2}-\tilde{\lambda}_{-}^{2}\right) 1_{x<0},
$$

and hence

$$
\int_{0}^{\infty}\left(e^{\psi(x) / 2}-1\right)^{2} \nu(d x)=C_{+} \int_{0}^{\infty} \frac{\left(e^{-\frac{\tilde{\lambda}_{+}^{2} x^{2}}{2}}-e^{-\frac{\lambda_{+}^{2} x^{2}}{2}}\right)^{2}}{x^{\alpha+1}} d x
$$

We can show that the right side of the above equation is finite. Using similar arguments, we can prove $\int_{-\infty}^{0}\left(e^{\psi(x) / 2}-1\right)^{2} \nu(d x)<\infty$. Thus, condition (A.5) holds if and only if $\alpha=\tilde{\alpha}, C_{+}=\tilde{C}_{+}$and, $C_{-}=\tilde{C}_{-}$.

Condition (A.6) holds if and only if

$$
\int_{|x| \leq 1} x(\tilde{\nu}-\nu)(d x)=\tilde{m}-\int_{|x|>1} x \tilde{\nu}(d x)-m+\int_{|x|>1} x \nu(d x),
$$

or

$$
\begin{aligned}
\tilde{m}-m & =\int_{-\infty}^{\infty} x(\tilde{\nu}-\nu)(d x) \\
& =2^{-\frac{\alpha+1}{2}} \Gamma\left(\frac{1-\alpha}{2}\right)\left(C_{+}\left(\tilde{\lambda}_{+}^{\alpha-1}-\lambda_{+}^{\alpha-1}\right)-C_{-}\left(\tilde{\lambda}_{-}^{\alpha-1}-\lambda_{-}^{\alpha-1}\right)\right) .
\end{aligned}
$$


Hence, $\mathbb{P}$ and $\mathbb{Q}$ are equivalent if and only if $\alpha=\tilde{\alpha}, C_{+}=\tilde{C}_{+}, C_{-}=\tilde{C}_{-}$and

$$
\tilde{m}-m=2^{-\frac{\alpha+1}{2}} \Gamma\left(\frac{1-\alpha}{2}\right)\left(C_{+}\left(\tilde{\lambda}_{+}^{\alpha-1}-\lambda_{+}^{\alpha-1}\right)-C_{-}\left(\tilde{\lambda}_{-}^{\alpha-1}-\lambda_{-}^{\alpha-1}\right)\right) .
$$

The Lévy triplet (3.5) can be obtained from (A.8) in Theorem A.3 with $\eta=0$.

\section{A.3 Proof of Proposition 3.10 and Proposition 3.11}

Lemma A.4. For $a \in \mathbb{R}_{+}$, the following equality holds

$$
\int_{\beta}^{\infty} s^{-a-1} e^{-s} d s=\beta^{-a-1} e^{-\beta}+o\left(\beta^{-a-1} e^{-\beta}\right)
$$

and

$$
\int_{\beta}^{\infty} s^{-a-1} e^{-\frac{s^{2}}{2}} d s=\beta^{-a-2} e^{-\frac{\beta^{2}}{2}}+o\left(\beta^{-a-2} e^{-\frac{\beta^{2}}{2}}\right)
$$

as $\beta \rightarrow \infty$.

Proof. By integration by parts, if $\beta>0$, we obtain

$$
\int_{\beta}^{\infty} s^{-a-1} e^{-s} d s=\beta^{-a-1} e^{-\beta}-(a+1) \int_{\beta}^{\infty} s^{-a-2} e^{-s} d s \leq \beta^{-a-1} e^{-\beta}
$$

and

$$
\begin{aligned}
\int_{\beta}^{\infty} s^{-a-1} e^{-s} d s & =\beta^{-a-1} e^{-\beta}-(a+1) \beta^{-a-2} e^{-\beta}+(a+1)(a+2) \int_{\beta}^{\infty} s^{-a-3} e^{-s} d s \\
& \geq \beta^{-a-1} e^{-\beta}-(a+1) \beta^{-a-2} e^{-\beta}
\end{aligned}
$$

when $\beta \rightarrow \infty$, the first result is proved. By integration by parts again, if $\beta>0$, we obtain

$$
\int_{\beta}^{\infty} s^{-a-1} e^{-\frac{s^{2}}{2}} d s=\beta^{-a-2} e^{-\frac{\beta^{2}}{2}}-(a+2) \int_{\beta}^{\infty} s^{-a-3} e^{-\frac{s^{2}}{2}} d s \leq \beta^{-a-2} e^{-\frac{\beta^{2}}{2}}
$$


and

$$
\begin{aligned}
& \int_{\beta}^{\infty} s^{-a-1} e^{-\frac{s^{2}}{2}} d s \\
& =\beta^{-a-2} e^{-\frac{\beta^{2}}{2}}-(a+2) \beta^{-a-4} e^{-\frac{\beta^{2}}{2}}+(a+2)(a+4) \int_{\beta}^{\infty} s^{-a-5} e^{-\frac{s^{2}}{2}} d s \\
& \geq \beta^{-a-2} e^{-\frac{\beta^{2}}{2}}-(a+2) \beta^{-a-4} e^{-\frac{\beta^{2}}{2}}
\end{aligned}
$$

when $\beta \rightarrow \infty$, the second result is proved.

We consider the following result:

Proposition A.5. Let $X$ be an infinitely divisible random variable in $\mathbb{R}$, with Lévy triplet $(\gamma, 0, \nu)$. Then we have

$$
\mathbb{P}(|X-m| \geq \lambda) \geq \frac{1}{4}(1-\exp (-\nu(u \in \mathbb{R}:|u| \geq 2 \lambda))), \quad \lambda>0 .
$$

for all $m \in \mathbb{R}$.

Proof. See Lemma 5.4 of Breton et al. (2007).

Taking into account Proposition A.5 and Lemma A.4, we can prove Proposition 3.10 and Proposition 3.11.

Proof of Proposition 3.10. By Chebyshev's Inequality, the upper bound part can be proved. Applying the following elementary fact

$$
1-\exp (-z) \sim z, \quad z \rightarrow 0
$$

and according to (A.13), we obtain

$$
\begin{aligned}
\mathbb{P}(|X-m| \geq \lambda) & \geq \frac{1}{4}\left(1-\exp \left[-C_{+} \int_{2 y}^{\infty} x^{-\alpha-1} e^{-\lambda_{+} x} d x-C_{-} \int_{2 y}^{\infty} x^{-\alpha-1} e^{-\lambda_{-} x} d x\right]\right) \\
& \sim \frac{1}{4}\left[C_{+} \int_{2 y}^{\infty} x^{-\alpha-1} e^{-\lambda_{+} x} d x+C_{-} \int_{2 y}^{\infty} x^{-\alpha-1} e^{-\lambda_{-} x} d x\right]|x|^{\alpha+1} e^{-\frac{2 \lambda}{|x|}} R(d x),
\end{aligned}
$$


as $y \rightarrow \infty$. By using (A.11) of Lemma A.4, we have

$$
\int_{2 y}^{\infty} x^{-\alpha-1} e^{-\lambda x} d x \sim(2 y)^{-\alpha-1} \lambda^{-1} e^{-2 \lambda y}
$$

Hence we obtain

$$
\mathbb{P}(|X-m| \geq \lambda) \geq K(2 y)^{-\alpha-1} \lambda^{-1} e^{-2 \lambda y}
$$

for some constant $K$ independent of $y$ and $\bar{\lambda}=\min \left\{\lambda_{+}, \lambda_{-}\right\}$as $y \rightarrow \infty$

Proof of Proposition 3.11. Using the same method in the proof of Proposition 3.10 with (A.12) of Lemma A.4, we can obtain the result. 


\section{References}

Andrews, L. D. (1998): Special Functions Of Mathematics For Engineers, Oxford University Press, 2nd ed.

Asmussen, S. and Glynn, P. (2007): Stochastic Simulation: Algorithms and Analysis, Springer.

Barndorff-Nielsen, O. E. and Shephard, N. (2001): Normal modified stable processes, Economics Series Working Papers from University of Oxford, Department of Economics, 72 .

Barone-Adesi, G., Engle, R., and Mancini, L. (2008): A GARCH option pricing model with filtered historical simulation, Review of Financial Studies, 21(3), 1223-1258.

Bianchi, M. L., Rachev, S. T., Kim, Y. S., and Fabozzi, F. J. (2008): Tempered infinitely divisible distributions and processes, Technical report, (http://www.statistik.unikarlsruhe.de/292.php).

Bollerslev, T. (1986): Generalized autoregressive conditional heteroskedasticity, Journal of Econometrics, 31, 307-327.

Boyarchenko, S. I. and Levendorskiı̌, S. Z. (2000): Option pricing for truncated Lévy processes, International Journal of Theoretical and Applied Finance, 3, 549-552.

Breton, J. C., Houdré, C., and Privault, N. (2007): Dimension free and infinite variance tail estimates on poisson space, Acta Applicandae Mathematicae, 95(3), 151-203.

Carr, P., Geman, H., Madan, D., and Yor, M. (2002): The fine structure of asset returns: An empirical investigation, Journal of Business, 75(2), 305-332.

Chernov, M. and Ghysels, E. (2000): A study towards a unified approach to the joint estimation of objective and risk neutral measures for the purpose of options valuation, Journal of Financial Economics, 56(3), 407-458. 
Christoffersen, P., Elkamhi, R., Feunou, B., and Jacobs, K. (2008): Option Valuation with Conditional Heteroskedasticity and Non-Normality, Tech. rep., McGill University.

Cont, R. and Tankov, P. (2004): Financial Modelling with Jump Processes, Chapman \& Hall / CRC.

Duan, J. (1999): Conditionally fat-tailed distributions and the volatility smile in options, Tech. rep., Hong Kong University of Science and Technology.

Duan, J. and Simonato, J. (1998): Empirical Martingale Simulation for Asset Prices, Management Science, 44(9), 1218-1233.

Duan, J.-C. (1995): The GARCH option pricing model, Mathematical Finance, 5(1), $13-32$.

Duan, J.-C., Ritchken, P., and Sun, Z. (2006): Approximating GARCH-jumps models, jump-diffusion processes, and option pricing, Mathematical Finance, 16(1), 21-52.

Engle, R. (1982): Autoregressive conditional heteroskedasticity with estimates of the variance of united kingdom inflation, Econometrica, 50, 987-1007.

Kim, Y. S. and Lee, J. H. (2006): The relative entropy in CGMY processes and its applications to finance, Mathematical Methods of Operations Research, 66(2), 327338.

Kim, Y. S., Rachev, S. T., Bianchi, M. L., and Fabozzi, F. J. (2008a): Financial market models with Lévy processes and time-varying volatility, Journal of Banking and Finance, 32, 1363-1378.

Kim, Y. S., Rachev, S. T., Bianchi, M. L., and Fabozzi, F. J. (2008b): A new tempered stable distribution and its application to finance, In G. Bol, S. T. Rachev, and R. Wuerth (Eds.), Risk Assessment: Decisions in Banking and Finance, Physika Verlag, Springer. 
Kim, Y. S., Rachev, S. T., Chung, D. M., and Bianchi, M. L. (2008c): The modified tempered stable distribution, GARCH-models and option pricing, Probability and Mathematical Statistics. To appear.

Koponen, I. (1995): Analytic approach to the problem of convergence of truncated Lévy flights towards the gaussian stochastic process, Physical Review E, 52, 1197-1199.

Marsaglia, G., Tsang, W., and Wang, G. (2003): Evaluating Kolmogorov's distribution, Journal of Statistical Software, 8(18).

Menn, C. and Rachev, S. T. (2005a). A GARCH option pricing model with $\alpha$-stable innovations, European Journal of Operational Research, 163, 201-209.

Menn, C. and Rachev, S. T. (2005b): Smoothly truncated stable distributions, GARCH-models, and option pricing, Technical report, (http://www.statistik.unikarlsruhe.de/292.php).

Poirot, J. and Tankov, P. (2006): Monte carlo option pricing for tempered stable (CGMY) processes, Asia-Pacific Finan Markets, 13, 327-344.

Rosiński, J. (2007): Tempering stable processes, Stochastic Processes and Their Applications, 117(6), 677-707.

Sato, K. (1999): Lévy Processes and Infinitely Divisible Distributions, Cambridge University Press.

Schoutens, W. (2003): Lévy Processes in Finance: Pricing Financial Derivatives, John Wiley and Sons.

Stentoft, L. (2008): American Option Pricing Using GARCH Models and the Normal Inverse Gaussian Distribution, Journal of Financial Econometrics, 6(4), 540-582. 


\section{Notes}

\footnotetext{
${ }^{1}$ See Andrews (1998).

${ }^{2}$ Kraft Foods (KFT) is excluded because the time series we employ begins in 1997 but this company was listed on 2001.
} 
Table 1: Estimated normal-GARCH parameters from October 1, 1997 to December 31, 2006 for 29 component companies of the DJIA index.

\begin{tabular}{|c|c|c|c|c|c|}
\hline & ticker & $\beta_{1}$ & $\alpha_{1}$ & $\alpha_{0}$ & $\lambda$ \\
\hline Alcoa Incorporated & AA & 0.9599 & 0.0338 & $2.6293 E-6$ & 0.0410 \\
\hline American Express Company & AXP & 0.9224 & 0.0731 & $2.1441 E-6$ & 0.0732 \\
\hline Boeing Corporation & $\mathrm{BA}$ & 0.9325 & 0.0572 & $5.1542 E-6$ & 0.0603 \\
\hline Bank of America Corporation & $\mathrm{BAC}$ & 0.9550 & 0.0416 & $1.2013 E-6$ & 0.0656 \\
\hline Citigroup Incorporated & $\mathrm{C}$ & 0.9577 & 0.0402 & $8.3005 E-7$ & 0.0795 \\
\hline Caterpillar Incorporated & CAT & 0.9824 & 0.0152 & $8.9119 E-7$ & 0.0626 \\
\hline Chevron & CVX & 0.9216 & 0.0625 & $3.9714 E-6$ & 0.0540 \\
\hline DuPont & $\mathrm{DD}$ & 0.9686 & 0.0293 & $5.6971 E-7$ & 0.0324 \\
\hline Walt Disney Company & DIS & 0.9041 & 0.0852 & $6.6607 E-6$ & 0.0471 \\
\hline General Electric Company & GE & 0.9606 & 0.0370 & $5.6093 E-7$ & 0.0627 \\
\hline General Motors Corporation & GM & 0.9228 & 0.0585 & $9.5254 E-6$ & 0.0275 \\
\hline Home Depot Incorporated & HD & 0.9620 & 0.0362 & $9.7257 E-7$ & 0.0675 \\
\hline Hewlett-Packard Company & HPQ & 0.9869 & 0.0111 & $1.4125 E-6$ & 0.0405 \\
\hline International Business Machines & IBM & 0.9179 & 0.0794 & $2.8849 E-6$ & 0.0658 \\
\hline Intel Corporation & INTC & 0.9699 & 0.0268 & $2.2101 E-6$ & 0.0529 \\
\hline Johnson\&Johnson & JNJ & 0.9181 & 0.0742 & $2.2397 E-6$ & 0.0548 \\
\hline JPMorgan Chase \& Company & JPM & 0.9432 & 0.0543 & $1.0285 E-6$ & 0.0617 \\
\hline Coca-Cola Company & $\mathrm{KO}$ & 0.9528 & 0.0439 & $9.0481 E-7$ & 0.0362 \\
\hline McDonald's Corporation & MCD & 0.9538 & 0.0407 & $1.8980 E-6$ & 0.0329 \\
\hline 3M Company & MMM & 0.8478 & 0.1034 & $1.3852 E-5$ & 0.0566 \\
\hline Merck \& Company, Incorpoarated & MRK & 0.9063 & 0.0221 & $2.6409 E-5$ & 0.0240 \\
\hline Microsoft Corporation & MSFT & 0.9348 & 0.0619 & $1.6078 E-6$ & 0.0644 \\
\hline Pfizer Incorporated & PFE & 0.8869 & 0.0887 & $1.0399 E-5$ & 0.0326 \\
\hline Procter and Gamble Company & PG & 0.9625 & 0.0360 & $3.0415 E-7$ & 0.0673 \\
\hline AT\&T Incorporated & $\mathrm{T}$ & 0.9356 & 0.0607 & $2.2891 E-6$ & 0.0253 \\
\hline United Technologies & UTX & 0.8934 & 0.0994 & $4.5332 E-6$ & 0.1027 \\
\hline Verizon Company & $\mathrm{VZ}$ & 0.9352 & 0.0614 & $1.4839 E-6$ & 0.0352 \\
\hline Wal-Mart Stores Incorporated & WMT & 0.9650 & 0.0335 & $4.8725 E-7$ & 0.0458 \\
\hline Exxon Mobil Corporation & XOM & 0.9336 & 0.0559 & $2.5577 E-6$ & 0.0602 \\
\hline
\end{tabular}


Table 2: Estimated parameters of the innovation processes for the CTS-GARCH and RDTS-GARCH models from October 1, 1997 to December 31, 2006 for 29 component companies of the DJIA index.

\begin{tabular}{|c|c|c|c|c|c|c|}
\hline \multirow[b]{2}{*}{ Ticker } & \multicolumn{3}{|c|}{ CTS } & \multicolumn{3}{|c|}{ RDTS } \\
\hline & $\alpha$ & $\lambda_{+}$ & $\lambda_{-}$ & $\alpha$ & $\lambda_{+}$ & $\lambda_{-}$ \\
\hline AA & 1.8499 & 0.3146 & 7.5000 & 1.8887 & 0.2024 & 10.5721 \\
\hline AXP & 1.7500 & 0.3805 & 9.7309 & 1.8803 & 0.2833 & 10.0845 \\
\hline BA & 1.7329 & 0.1753 & 0.5522 & 1.7461 & 0.1608 & 0.4295 \\
\hline BAC & 1.7441 & 0.6178 & 0.4130 & 1.7325 & 0.4828 & 0.3444 \\
\hline $\mathrm{C}$ & 1.7376 & 0.2255 & 0.9701 & 1.7430 & 0.1736 & 0.6844 \\
\hline CAT & 1.7325 & 0.2531 & 1.7459 & 1.7325 & 0.2305 & 1.0083 \\
\hline CVX & 1.7637 & 0.6729 & 2.0788 & 1.7512 & 0.4769 & 1.1658 \\
\hline $\mathrm{DD}$ & 1.9220 & 0.0359 & 7.4336 & 1.9322 & 0.1916 & 11.2821 \\
\hline DIS & 1.7325 & 0.1691 & 1.0917 & 1.7325 & 0.1580 & 0.7457 \\
\hline GE & 1.8965 & 0.4004 & 7.5000 & 1.9195 & 0.2970 & 7.5151 \\
\hline GM & 1.8545 & 0.0497 & 2.8984 & 1.7336 & 0.1302 & 1.2418 \\
\hline HD & 1.7500 & 0.2357 & 4.6684 & 1.7752 & 0.1910 & 1.8216 \\
\hline HPQ & 1.7325 & 0.0751 & 0.4124 & 1.7325 & 0.0762 & 0.3574 \\
\hline IBM & 1.7325 & 0.1098 & 0.5483 & 1.7325 & 0.1098 & 0.4406 \\
\hline INTC & 1.8234 & 0.2091 & 9.9281 & 1.9999 & 0.1784 & 9.9591 \\
\hline JNJ & 1.8322 & 0.2714 & 4.2282 & 1.7689 & 0.2309 & 1.4297 \\
\hline JPM & 1.7473 & 0.5283 & 3.0839 & 1.7325 & 0.3847 & 1.2420 \\
\hline $\mathrm{KO}$ & 1.7535 & 0.2020 & 7.8378 & 1.7812 & 0.1566 & 5.7200 \\
\hline MCD & 1.7325 & 0.1670 & 0.5328 & 1.7325 & 0.1654 & 0.4167 \\
\hline MMM & 1.7325 & 0.1249 & 0.7565 & 1.7325 & 0.1230 & 0.5714 \\
\hline MRK & 1.7325 & 0.1158 & 0.1265 & 1.7325 & 0.1163 & 0.1257 \\
\hline MSFT & 1.8710 & 0.1293 & 6.0573 & 1.8547 & 0.1427 & 2.5893 \\
\hline PFE & 1.7402 & 0.3854 & 1.6620 & 1.7591 & 0.3072 & 1.0720 \\
\hline PG & 1.7325 & 0.2770 & 1.2074 & 1.7340 & 0.2530 & 0.7940 \\
\hline $\mathrm{T}$ & 1.7325 & 0.1619 & 0.4918 & 1.7325 & 0.1582 & 0.3912 \\
\hline UTX & 1.8077 & 0.1455 & 2.3654 & 1.7500 & 0.1931 & 1.2059 \\
\hline $\mathrm{VZ}$ & 1.8338 & 0.2310 & 5.5116 & 1.8431 & 0.1926 & 3.0450 \\
\hline WMT & 1.7325 & 0.4049 & 1.7809 & 1.7327 & 0.3118 & 1.0097 \\
\hline $\mathrm{XOM}$ & 1.7632 & 0.4682 & 0.8831 & 1.8285 & 0.2816 & 0.5421 \\
\hline
\end{tabular}


Table 3: Statistic of the goodness of fit tests

\begin{tabular}{|c|c|c|c|c|}
\hline Ticker & Model & KS & $p$-value & $\mathrm{AD}$ \\
\hline \multirow[t]{3}{*}{$\mathrm{AA}$} & Normal-GARCH & 0.0285 & 0.0340 & 1.3952 \\
\hline & CTS-GARCH & 0.0230 & 0.1408 & 0.1938 \\
\hline & RDTS-GARCH & 0.0230 & 0.1402 & 0.1948 \\
\hline \multirow[t]{3}{*}{$\overline{\mathrm{AXP}}$} & Normal-GARCH & 0.0249 & 0.0886 & 84.0733 \\
\hline & CTS-GARCH & 0.0144 & 0.6748 & 0.1090 \\
\hline & RDTS-GARCH & 0.0145 & 0.6655 & 0.2233 \\
\hline \multirow[t]{3}{*}{ BA } & Normal-GARCH & 0.0308 & 0.0173 & 15.6383 \\
\hline & CTS-GARCH & 0.0202 & 0.2575 & 0.0698 \\
\hline & RDTS-GARCH & 0.0196 & 0.2884 & 0.0850 \\
\hline \multirow[t]{3}{*}{$\mathrm{BAC}$} & Normal-GARCH & 0.0266 & 0.0240 & 0.3805 \\
\hline & CTS-GARCH & 0.0144 & 0.5390 & 0.0359 \\
\hline & RDTS-GARCH & 0.0138 & 0.5918 & 0.0639 \\
\hline \multirow[t]{3}{*}{$\mathrm{C}$} & Normal-GARCH & 0.0298 & 0.0073 & 160.1880 \\
\hline & CTS-GARCH & 0.0205 & 0.1400 & 0.1798 \\
\hline & RDTS-GARCH & 0.0215 & 0.1072 & 0.4389 \\
\hline \multirow[t]{3}{*}{ CAT } & Normal-GARCH & 0.0319 & 0.0124 & 1.9053 \\
\hline & CTS-GARCH & 0.0248 & 0.0914 & 0.1492 \\
\hline & RDTS-GARCH & 0.0245 & 0.0995 & 0.1406 \\
\hline \multirow[t]{3}{*}{ CVX } & Normal-GARCH & 0.0177 & 0.4113 & 0.1066 \\
\hline & CTS-GARCH & 0.0143 & 0.6843 & 0.0950 \\
\hline & RDTS-GARCH & 0.0135 & 0.7535 & 0.0970 \\
\hline \multirow[t]{3}{*}{ DD } & Normal-GARCH & 0.0354 & 0.0037 & 1.4710 \\
\hline & CTS-GARCH & 0.0284 & 0.0347 & 0.1104 \\
\hline & RDTS-GARCH & 0.0344 & 0.0053 & 0.1773 \\
\hline \multirow[t]{3}{*}{ DIS } & Normal-GARCH & 0.0381 & 0.0014 & 281.9976 \\
\hline & CTS-GARCH & 0.0265 & 0.0592 & 0.1241 \\
\hline & RDTS-GARCH & 0.0262 & 0.0649 & 0.2034 \\
\hline \multirow[t]{3}{*}{ GE } & Normal-GARCH & 0.0243 & 0.1033 & 0.3035 \\
\hline & CTS-GARCH & 0.0187 & 0.3436 & 0.1701 \\
\hline & RDTS-GARCH & 0.0188 & 0.3364 & 0.1782 \\
\hline \multirow[t]{3}{*}{$\overline{G M}$} & Normal-GARCH & 0.0428 & 0.0002 & 17852.7859 \\
\hline & CTS-GARCH & 0.0197 & 0.2837 & 0.1788 \\
\hline & RDTS-GARCH & 0.0211 & 0.2131 & 0.1993 \\
\hline \multirow[t]{3}{*}{$\mathrm{HD}$} & Normal-GARCH & 0.0338 & 0.0066 & 1.2829 \\
\hline & CTS-GARCH & 0.0126 & 0.8194 & 0.1547 \\
\hline & RDTS-GARCH & 0.0120 & 0.8620 & 0.1452 \\
\hline \multirow[t]{3}{*}{ HPQ } & Normal-GARCH & 0.0506 & 0.0000 & 3476.9698 \\
\hline & CTS-GARCH & 0.0205 & 0.2438 & 0.0810 \\
\hline & RDTS-GARCH & 0.0213 & 0.2072 & 0.0986 \\
\hline \multirow[t]{3}{*}{ IBM } & Normal-GARCH & 0.0554 & 0.0000 & 99.1506 \\
\hline & CTS-GARCH & 0.0219 & 0.1797 & 0.0962 \\
\hline & RDTS-GARCH & 0.0222 & 0.1682 & 0.0940 \\
\hline \multirow[t]{3}{*}{ INTC } & Normal-GARCH & 0.0266 & 0.0579 & 17.7457 \\
\hline & CTS-GARCH & 0.0160 & 0.5435 & 0.1512 \\
\hline & RDTS-GARCH & 0.0266 & 0.0580 & 5.7968 \\
\hline
\end{tabular}


(Continue)

\begin{tabular}{|c|c|c|c|c|}
\hline Ticker & Model & $\mathrm{KS}$ & $p$-value & $\mathrm{AD}$ \\
\hline \multirow[t]{3}{*}{ JNJ } & Normal-GARCH & 0.0405 & 0.0005 & 0.7086 \\
\hline & CTS-GARCH & 0.0262 & 0.0647 & 0.1181 \\
\hline & RDTS-GARCH & 0.0201 & 0.2626 & 0.1180 \\
\hline \multirow[t]{3}{*}{ JPM } & Normal-GARCH & 0.0323 & 0.0009 & 1.3883 \\
\hline & CTS-GARCH & 0.0191 & 0.1363 & 0.1826 \\
\hline & RDTS-GARCH & 0.0182 & 0.1727 & 0.1733 \\
\hline \multirow[t]{3}{*}{$\mathrm{KO}$} & Normal-GARCH & 0.0379 & 0.0015 & 1.2765 \\
\hline & CTS-GARCH & 0.0146 & 0.6633 & 0.1520 \\
\hline & RDTS-GARCH & 0.0151 & 0.6168 & 0.1476 \\
\hline \multirow[t]{3}{*}{ MCD } & Normal-GARCH & 0.0403 & 0.0006 & 5.9558 \\
\hline & CTS-GARCH & 0.0110 & 0.9215 & 0.0577 \\
\hline & RDTS-GARCH & 0.0119 & 0.8678 & 0.0765 \\
\hline \multirow[t]{3}{*}{ MMM } & Normal-GARCH & 0.0503 & 0.0000 & 1.1493 \\
\hline & CTS-GARCH & 0.0165 & 0.5057 & 0.0861 \\
\hline & RDTS-GARCH & 0.0167 & 0.4876 & 0.0918 \\
\hline \multirow[t]{3}{*}{ MRK } & Normal-GARCH & 0.0535 & 0.0000 & 1334.3847 \\
\hline & CTS-GARCH & 0.0147 & 0.6519 & 0.0354 \\
\hline & RDTS-GARCH & 0.0146 & 0.6606 & 0.0394 \\
\hline \multirow[t]{3}{*}{ MSFT } & Normal-GARCH & 0.0379 & 0.0015 & 2.2925 \\
\hline & CTS-GARCH & 0.0210 & 0.2170 & 0.2039 \\
\hline & RDTS-GARCH & 0.0198 & 0.2786 & 0.2402 \\
\hline \multirow[t]{3}{*}{ PFE } & Normal-GARCH & 0.0233 & 0.1328 & 0.9711 \\
\hline & CTS-GARCH & 0.0161 & 0.5332 & 0.1184 \\
\hline & RDTS-GARCH & 0.0162 & 0.5262 & 0.1326 \\
\hline \multirow[t]{3}{*}{ PG } & Normal-GARCH & 0.0277 & 0.0428 & 1.6565 \\
\hline & CTS-GARCH & 0.0115 & 0.8959 & 0.1423 \\
\hline & RDTS-GARCH & 0.0116 & 0.8873 & 0.1601 \\
\hline \multirow[t]{3}{*}{$\mathrm{T}$} & Normal-GARCH & 0.0341 & 0.0000 & 36014.5914 \\
\hline & CTS-GARCH & 0.0121 & 0.4690 & 0.1089 \\
\hline & RDTS-GARCH & 0.0128 & 0.3976 & 0.2522 \\
\hline \multirow[t]{3}{*}{ UTX } & Normal-GARCH & 0.0456 & 0.0001 & 0.0990 \\
\hline & CTS-GARCH & 0.0209 & 0.2240 & 0.0640 \\
\hline & RDTS-GARCH & 0.0193 & 0.3109 & 0.0535 \\
\hline \multirow[t]{3}{*}{ VZ } & Normal-GARCH & 0.0383 & 0.0013 & 0.3669 \\
\hline & CTS-GARCH & 0.0219 & 0.1807 & 0.1478 \\
\hline & RDTS-GARCH & 0.0215 & 0.1973 & 0.1528 \\
\hline \multirow[t]{3}{*}{ WMT } & Normal-GARCH & 0.0257 & 0.0729 & 0.2594 \\
\hline & CTS-GARCH & 0.0121 & 0.8568 & 0.0650 \\
\hline & RDTS-GARCH & 0.0116 & 0.8878 & 0.0657 \\
\hline \multirow[t]{3}{*}{ XOM } & Normal-GARCH & 0.0232 & 0.1361 & 0.5962 \\
\hline & CTS-GARCH & 0.0122 & 0.8508 & 0.0611 \\
\hline & RDTS-GARCH & 0.0122 & 0.8500 & 0.0593 \\
\hline
\end{tabular}


Table 4: DJIA index estimated normal-GARCH parameters from January 2, 1996 to any Wednesday from January 4, 2006 to June 6, 2007. Dates are in the form yyyymmdd.

\begin{tabular}{|c|c|c|c|c|}
\hline Date & $\beta_{1}$ & 1 & $\alpha_{0}$ & $\lambda$ \\
\hline 4 & 3 & 921 & & \\
\hline & & & & \\
\hline & & & & \\
\hline & & & & \\
\hline & & & & \\
\hline & & & & \\
\hline & & & & \\
\hline & & & & \\
\hline & & & & \\
\hline & & & & \\
\hline & & & & \\
\hline & & & & \\
\hline & & & & \\
\hline & & & & \\
\hline & & & & \\
\hline & & & & \\
\hline & & & & \\
\hline & & & & \\
\hline & & & & \\
\hline & & & & \\
\hline & & & & \\
\hline & & & & \\
\hline & & & & \\
\hline & & & & \\
\hline & & & & \\
\hline & & & & \\
\hline & & & & \\
\hline & & & & \\
\hline & & & & \\
\hline & & & & \\
\hline & & & & \\
\hline & & & & \\
\hline & & & 0.000 & 0165 \\
\hline & & & & \\
\hline & & & & \\
\hline & & & & \\
\hline & & & & \\
\hline & & & & \\
\hline 27 & 0.90 & & 0.0000 & 9289 \\
\hline & & & & 1208 \\
\hline & & & & \\
\hline & & & & \\
\hline & & & & \\
\hline & & & & \\
\hline 0061108 & 0.908256665 & 0.082214211 & 0.000001230 & 0.0634031 \\
\hline
\end{tabular}




\begin{tabular}{lllll} 
(Continue) & & & & \\
\hline 20061115 & 0.908603981 & 0.082163206 & 0.000001200 & 0.063636590 \\
20061122 & 0.909216905 & 0.082012625 & 0.000001145 & 0.064181416 \\
20061129 & 0.908643826 & 0.081862611 & 0.000001223 & 0.063137579 \\
20061206 & 0.909327202 & 0.081524806 & 0.000001185 & 0.063215486 \\
20061213 & 0.910077129 & 0.081194900 & 0.000001129 & 0.063372442 \\
20061220 & 0.910395984 & 0.081110766 & 0.000001101 & 0.064276960 \\
20061227 & 0.910170083 & 0.081188649 & 0.000001118 & 0.064276967 \\
20070103 & 0.910728597 & 0.080880770 & 0.000001086 & 0.064049842 \\
20070110 & 0.911259701 & 0.080646861 & 0.000001049 & 0.063648369 \\
20070117 & 0.911677497 & 0.080467826 & 0.000001019 & 0.064199176 \\
20070124 & 0.911109046 & 0.080910692 & 0.000001041 & 0.063985713 \\
20070131 & 0.911345423 & 0.080545067 & 0.000001045 & 0.064172538 \\
20070207 & 0.912454661 & 0.079996173 & 0.000000978 & 0.064213568 \\
20070214 & 0.912293093 & 0.080054217 & 0.000000989 & 0.064578837 \\
20070221 & 0.912878969 & 0.079749900 & 0.000000955 & 0.063792703 \\
20070228 & 0.907712192 & 0.080889040 & 0.000001417 & 0.062291435 \\
20070307 & 0.908990823 & 0.079857765 & 0.000001381 & 0.061764192 \\
20070314 & 0.909243931 & 0.079590626 & 0.000001385 & 0.061167423 \\
20070321 & 0.909385517 & 0.079234126 & 0.000001386 & 0.061954361 \\
20070328 & 0.909744245 & 0.079098044 & 0.000001365 & 0.061544456 \\
20070404 & 0.909621305 & 0.079172161 & 0.000001362 & 0.062314976 \\
20070411 & 0.909290646 & 0.079480096 & 0.000001362 & 0.062107768 \\
20070418 & 0.908848424 & 0.079885589 & 0.000001364 & 0.063504981 \\
20070425 & 0.909236929 & 0.079510540 & 0.000001361 & 0.064247765 \\
20070502 & 0.909228228 & 0.079572615 & 0.000001349 & 0.065073633 \\
20070509 & 0.908949832 & 0.080007948 & 0.000001331 & 0.065964853 \\
20070516 & 0.909345811 & 0.079531912 & 0.000001338 & 0.065845470 \\
20070523 & 0.909494162 & 0.079563098 & 0.000001314 & 0.066272392 \\
20070530 & 0.909527828 & 0.079539061 & 0.000001312 & 0.066581613 \\
20070606 & 0.909457567 & 0.079615742 & 0.000001313 & 0.065829470 \\
\hline Average & 0.907736375 & 0.081812917 & 0.000001351 & 0.061523578 \\
\hline & & & &
\end{tabular}


Table 5: DJIA market parameters of the innovation processes for the CTS-GARCH and RDTS-GARCH models. The DJIA time series from January 2, 1996 to any Wednesday from January 4, 2006 to June 6, 2007 are considered.

\begin{tabular}{|c|c|c|c|c|c|c|c|c|}
\hline \multirow[b]{2}{*}{ Date } & \multicolumn{4}{|c|}{ CTS } & \multicolumn{4}{|c|}{ RDTS } \\
\hline & C & $\lambda_{-}$ & $\lambda_{+}$ & $\alpha$ & C & $\lambda_{+}$ & $\lambda_{-}$ & $\alpha$ \\
\hline 60104 & 0.1145 & 14 & 1.0346 & 1.7613 & 0 & 36 & 905 & נת \\
\hline & 60 & 0.3354 & 1.0 & & 92 & & 0.2922 & \\
\hline 060118 & 0.1135 & 0.3293 & 1.0361 & 1.7634 & 0.0781 & 0.9453 & 0.2894 & 1.8206 \\
\hline 060125 & 0.1248 & 0.3523 & 1.0329 & 1.7400 & 0.0836 & 0.9349 & 0.2996 & 1. \\
\hline 60201 & 232 & 0.3474 & 1.0461 & & 0.0831 & 9473 & 0.2982 & 1.8076 \\
\hline 060208 & 1203 & 0.3402 & 1.0428 & & 0.0819 & 9472 & 0.2952 & 18106 \\
\hline 060215 & 0.1204 & 0.3403 & 1.0448 & 1.7491 & & & & 1.8103 \\
\hline 060222 & 0.1207 & 0.3407 & 1.0474 & & 22 & 11 & & 1.8100 \\
\hline 060301 & 0.1200 & 0.3395 & 1.0497 & & 0.0818 & 0.9529 & 0.2949 & 1.8111 \\
\hline 60308 & 1210 & 0.3417 & 1.0419 & & 0. & 0.9459 & 0.2 & \\
\hline 0315 & 196 & 0.3378 & 1.0 & & 0.0818 & 74 & 0.2 & 10 \\
\hline 22 & 201 & 0.3393 & 1.0 & & 20 & & 3 & \\
\hline 060329 & 0.1190 & 0.3369 & 1.0600 & 24 & 0.0815 & & 02 & 1 \\
\hline 105 & 0.1193 & 0.3381 & 1.0 & & & & & \\
\hline 060412 & 0.1185 & 0.3361 & 1.0 & & 0.0811 & & & \\
\hline & & 0.3530 & 0. & & & & & \\
\hline & 39 & 0.3544 & 0.9 & & 22 & 11 & 1 & \\
\hline 503 & 1247 & 0.3561 & 0.9 & & & & & \\
\hline & & 0.3568 & 0.9 & & & & & \\
\hline 060517 & 0.1338 & 0.3801 & 1.0156 & 22 & 0.0860 & 94 & 0.3091 & 1.7996 \\
\hline 0060524 & 0.1341 & 0.3801 & 1.0057 & & & & & \\
\hline & & & & & & & & \\
\hline & 90 & 0.39 & 1.0 & & 79 & 1 & 0. & \\
\hline 514 & & 0.38 & 1.0 & & 57 & & & \\
\hline 21 & 0. & 0.3829 & 1.0 & & 0. & & & \\
\hline 20060628 & 0.1341 & 0.3820 & 1.0246 & 22 & 0.0859 & 54 & 0.3 & 1.8002 \\
\hline 20060705 & 0.1327 & 0.3795 & 1.0093 & & 0.0854 & & & 1.8013 \\
\hline & & & & & & & & \\
\hline & 01 & 0.3753 & 1.0 & & 0.0840 & 4 & 0.3 & \\
\hline & 82 & 0.3706 & 1.0 & & 0. & & & \\
\hline 02 & 0.1283 & 0.3702 & 1.0 & & 0 & & & \\
\hline 20060809 & 0.1288 & 0.3721 & 0.9934 & 1.7319 & 0.0835 & 0.8960 & 0.3050 & 1.8060 \\
\hline & & & & & & & & 1.8065 \\
\hline & & & & & & & & \\
\hline & & 7 & 0.9 & & 0.0 & 2 & 0.3 & 1.8 \\
\hline 06 & 36 & 0.38 & 0.9 & 27 & 0.0 & 39 & 0.3 & 1.8 \\
\hline 0913 & & 0.3806 & 1.0 & & 0.0848 & 18 & 5 & 1.8 \\
\hline 20060920 & 0.1338 & 0.3854 & 1.0060 & 1.7225 & 0.0856 & 0.9008 & 0.3116 & 1.8007 \\
\hline 20060927 & 0.1315 & 0.3799 & 1.0127 & & 0.0848 & 0.9079 & 0.3097 & 1.8031 \\
\hline & & 0.3772 & & & 0.0845 & 0.9098 & & 1.8040 \\
\hline & & & & & & & & \\
\hline & & & & & 0.0856 & & & \\
\hline & & 0 & & 7228 & 0.0858 & & $0 ?$ & 1.80 \\
\hline 20061101 & 0.1335 & 0.3868 & 1.0077 & 1.7236 & 0.0854 & 0.9020 & 0.3126 & 1.8015 \\
\hline 20061108 & 0.1326 & 0.3847 & 1.0059 & 1.7252 & 0.0851 & 0.9017 & 0.3119 & 1.8021 \\
\hline 20061115 & 0.1323 & 0.3837 & 1.0112 & 1.7260 & 0.0851 & 0.9061 & 0.3117 & 1.8024 \\
\hline & & 3903 & & & 0.08 & 56 & 0.3142 & 1.80 \\
\hline & & & & & & & & \\
\hline 20061206 & 0.1398 & 0.4005 & 1.0249 & 1.7115 & 0.0882 & 0.9105 & 0.3188 & 1.7944 \\
\hline
\end{tabular}




\begin{tabular}{lllllllll} 
(Continue) & \multicolumn{1}{c}{ (1.0232 } & 1.7056 & 0.0891 & 0.9057 & 0.3218 & 1.7919 \\
\hline 20061213 & 0.1428 & 0.4090 & 1.0269 & 1.7037 & 0.0896 & 0.9078 & 0.3229 & 1.7909 \\
20061220 & 0.1439 & 0.4115 & 1.0269 & 1.7127 & 0.0881 & 0.9147 & 0.3192 & 1.7949 \\
20061227 & 0.1393 & 0.4003 & 1.0301 & 1.719 \\
20070103 & 0.1402 & 0.4027 & 1.0253 & 1.7109 & 0.0883 & 0.9099 & 0.3199 & 1.7942 \\
20070110 & 0.1420 & 0.4077 & 1.0258 & 1.7075 & 0.0888 & 0.9081 & 0.3214 & 1.7930 \\
20070117 & 0.1432 & 0.4104 & 1.0337 & 1.7053 & 0.0893 & 0.9129 & 0.3226 & 1.7918 \\
20070124 & 0.1420 & 0.4066 & 1.0377 & 1.7077 & 0.0890 & 0.9178 & 0.3214 & 1.7926 \\
20070131 & 0.1424 & 0.4087 & 1.0464 & 1.7075 & 0.0889 & 0.9239 & 0.3221 & 1.7931 \\
20070207 & 0.1454 & 0.4164 & 1.0471 & 1.7016 & 0.0899 & 0.9209 & 0.3248 & 1.7904 \\
20070214 & 0.1406 & 0.4046 & 1.0454 & 1.7110 & 0.0884 & 0.9245 & 0.3210 & 1.7945 \\
20070221 & 0.1422 & 0.4085 & 1.0433 & 1.7077 & 0.0889 & 0.9210 & 0.3221 & 1.7929 \\
20070228 & 0.1123 & 0.2817 & 0.9029 & 1.7566 & 0.0799 & 0.8606 & 0.2542 & 1.8108 \\
20070307 & 0.1102 & 0.2756 & 0.8981 & 1.7609 & 0.0791 & 0.8590 & 0.2513 & 1.8129 \\
20070314 & 0.1144 & 0.2857 & 0.9087 & 1.7522 & 0.0809 & 0.8639 & 0.2557 & 1.8083 \\
20070321 & 0.1145 & 0.2869 & 0.9191 & 1.7525 & 0.0808 & 0.8709 & 0.2564 & 1.8086 \\
20070328 & 0.1140 & 0.2848 & 0.9075 & 1.7531 & 0.0807 & 0.8631 & 0.2552 & 1.8088 \\
20070404 & 0.1153 & 0.2877 & 0.9110 & 1.7505 & 0.0812 & 0.8648 & 0.2564 & 1.8073 \\
20070411 & 0.1161 & 0.2900 & 0.9067 & 1.7486 & 0.0815 & 0.8606 & 0.2572 & 1.8065 \\
20070418 & 0.1170 & 0.2917 & 0.9185 & 1.7470 & 0.0819 & 0.8694 & 0.2581 & 1.8056 \\
20070425 & 0.1150 & 0.2867 & 0.9219 & 1.7512 & 0.0813 & 0.8741 & 0.2565 & 1.8074 \\
20070502 & 0.1157 & 0.2881 & 0.9237 & 1.7500 & 0.0815 & 0.8750 & 0.2570 & 1.8068 \\
20070509 & 0.1179 & 0.2937 & 0.9263 & 1.7452 & 0.0824 & 0.8752 & 0.2591 & 1.8044 \\
20070516 & 0.1183 & 0.2943 & 0.9430 & 1.7449 & 0.0826 & 0.8879 & 0.2596 & 1.8043 \\
20070523 & 0.1194 & 0.2970 & 0.9382 & 1.7425 & 0.0830 & 0.8834 & 0.2605 & 1.8031 \\
20070530 & 0.1180 & 0.2933 & 0.9445 & 1.7456 & 0.0825 & 0.8894 & 0.2592 & 1.8046 \\
20070606 & 0.1180 & 0.2939 & 0.9435 & 1.7457 & 0.0823 & 0.8887 & 0.2591 & 1.8050 \\
\hline Average & 0.1278 & 0.3574 & 1.0032 & 1.7330 & 0.0842 & 0.9095 & 0.2975 & 1.8037 \\
\hline
\end{tabular}

Table 6: Goodness of fit statistics. KS, AD, and $\chi^{2}$ with the relative $p$-value for the normal-GARCH, CTS-GARCH and RDTS-GARCH models from January 2, 1996 to any Wednesday from January 4, 2006 to June 6, 2007. Values are in average.

\begin{tabular}{lccc}
\hline & $\mathrm{KS}$ & $\mathrm{AD}$ & $\chi^{2}(p$-value $)$ \\
\hline Normal-GARCH & 0.0347 & 14.5832 & $185.0790(0.0016)$ \\
CTS-GARCH & 0.0327 & 0.0689 & $149.7951(0.0732)$ \\
RDTS-GARCH & 0.0328 & 0.0694 & $151.2415(0.0569)$ \\
\hline
\end{tabular}



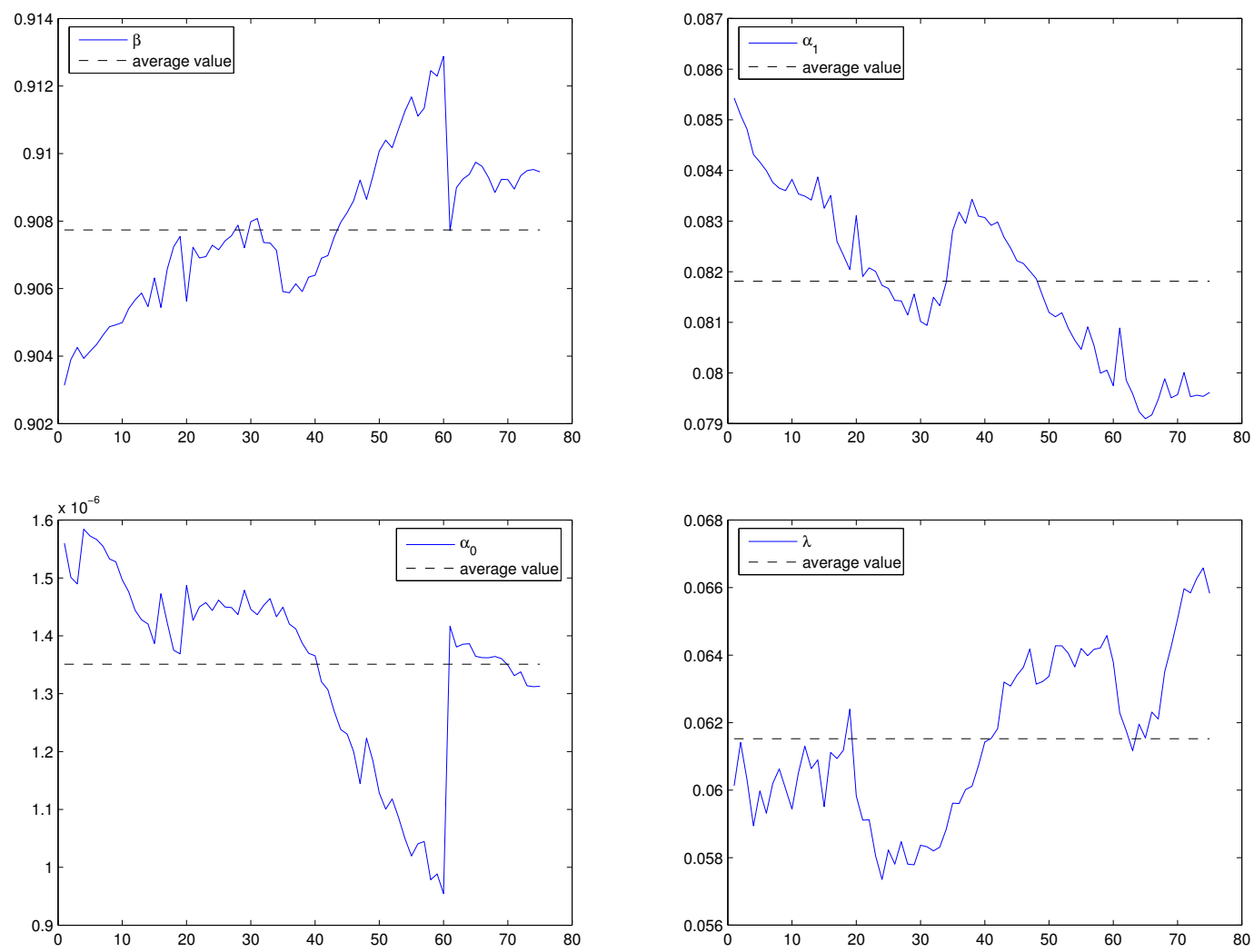

Figure 1: DJIA estimated market parameters for the normal-GARCH model from January 2, 1996 to any Wednesday from January 4, 2006 to June 6, 2007. 

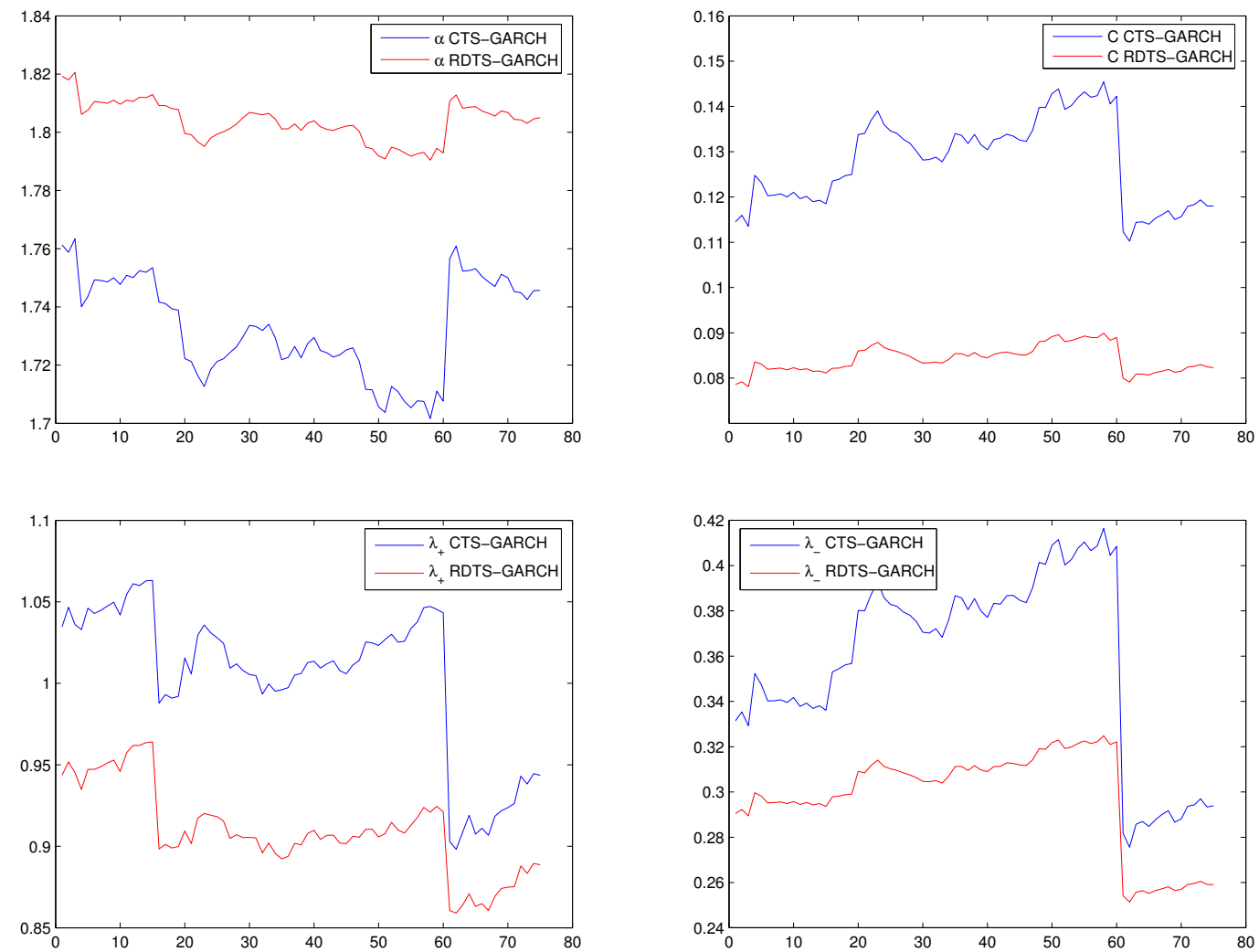

Figure 2: DJIA estimated market parameters for the CTS-GARCH and RDTS-GARCH models from January 2, 1996 to any Wednesday from January 4, 2006 to June 6, 2007. 

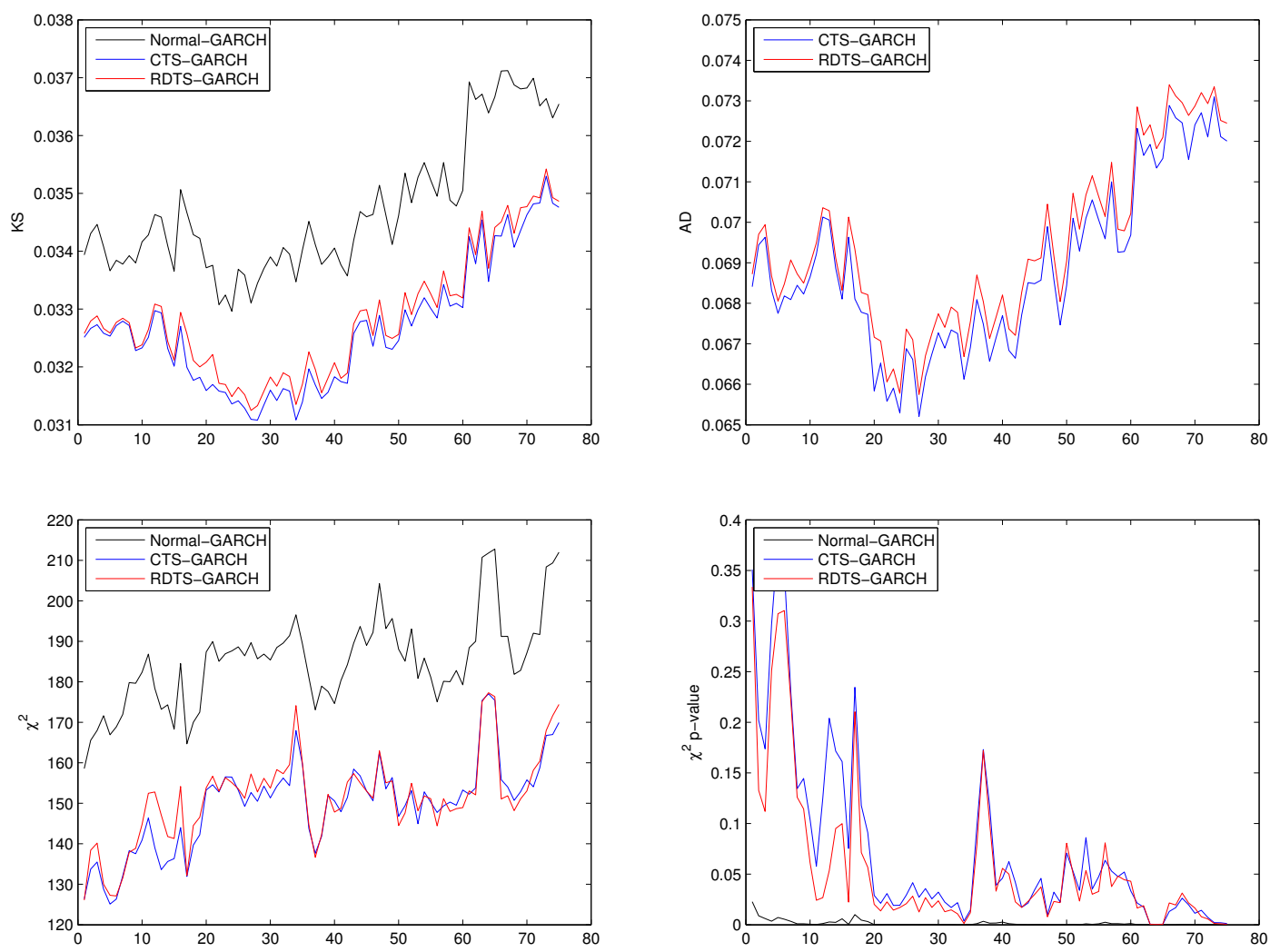

Figure 3: Goodness of fit. $\mathrm{KS}, \mathrm{AD}$ and $\chi^{2}$ with the relative $p$-value for the normalGARCH, CTS-GARCH and RDTS-GARCH models from January 2, 1996 to any Wednesday from January 4, 2006 to June 6, 2007. The AD statistic for the normalGARCH is not comparable, since it is always greater than 9.1474 . 
Table 7: Option pricing performance for 17 selected Wednesday (one per month) between January 4, 2006 and May 9, 2007.

\begin{tabular}{|c|c|c|c|c|c|}
\hline & & APE & $\mathrm{AAE}$ & RMSE & ARPE \\
\hline rmal-GARCH & 20060111 & 0.0988 & 0.6381 & 0.8394 & 0.6289 \\
\hline TS-GARCH & & 0.0489 & 51 & 4268 & .2524 \\
\hline RDTS-GARCH & & 0.0470 & 0.3035 & 0.4417 & 0.1441 \\
\hline ormal-GARCH & 20060208 & 0.1069 & 0.6513 & 0.7851 & 0.9685 \\
\hline TS-GARCH & & 0.0540 & 0.3292 & 4139 & 0.3883 \\
\hline RDTS-GARCH & & 0.0486 & 0.2958 & 0.4157 & 0.1733 \\
\hline rmal-GARCH & 20060315 & 0.0489 & 0.4487 & 0.5815 & 0.3760 \\
\hline TS-GARCH & & 0.0253 & 0.2321 & 0.3209 & 0.1286 \\
\hline RDTS-GARCH & & 0.0238 & 0.2188 & 0.3230 & 0.0513 \\
\hline Normal-GARCH & 20060412 & 0.0731 & 0.5483 & 0.7627 & 0.4077 \\
\hline TS-GARCH & & 0.0363 & 0.2726 & 4462 & 0.1401 \\
\hline RDTS-GARCH & & 0.0355 & 0.2661 & 0.4678 & 0.0816 \\
\hline ormal-GARCH & 2006 & 0.0591 & 0.6099 & 0.7897 & 0.5024 \\
\hline S-GARCH & & 0.0347 & & 4700 & 0.1747 \\
\hline RDTS-GARCH & & 0.0316 & 0.3260 & 0.4583 & 0.0739 \\
\hline rmal-GARCH & 2006 & 0.0510 & 0.3229 & 0.4081 & 0.9875 \\
\hline $\mathrm{CH}$ & & 0.0 & 0.2913 & & 0.3488 \\
\hline RDTS-GARCH & & 0.0548 & 0.3470 & 0.4563 & 0.1981 \\
\hline ormal-GARCH & 2006 & 0.0802 & 0.5860 & 0.7886 & 1.1047 \\
\hline $\mathrm{CH}$ & & 0.0284 & 0.2076 & & .3963 \\
\hline RDTS-GARCH & & 0.0258 & 0.1881 & 0.3268 & 0.1966 \\
\hline GARCH & 2006 & 0. & & & 0.7688 \\
\hline $\mathrm{CH}$ & & 0.02 & 38 & 57 & 0.2580 \\
\hline RDTS-GARCH & & 0.0282 & 0.2164 & 0.3342 & 0.1666 \\
\hline GARCH & 2006 & 0.0541 & 0.4947 & & 0.6993 \\
\hline $\mathrm{CH}$ & & 0.0293 & 577 & 18 & .3047 \\
\hline RDTS-GARCH & & 0.0248 & 0.2268 & 0.3602 & 0.1429 \\
\hline rmal-GARCH & 2006 & 76 & 84 & 78 & 4608 \\
\hline & & & & & 1791 \\
\hline RDTS-GARCH & & 0.0228 & 0.2527 & 0.4453 & 0.0917 \\
\hline Normal-GARCH & 20061 & 32 & 06 & 1 & .7525 \\
\hline & & & & & 3714 \\
\hline RDTS-GARCH & & 0.0615 & 0.4297 & 0.5800 & 0.1842 \\
\hline ormol $\mathrm{CARCH}$ & 2006 & 1 & 1 & 4 & 3599 \\
\hline & & & & & 0.1637 \\
\hline RDTS-GARCH & & 0.0176 & 0.2196 & 0.3473 & 0.0747 \\
\hline & & & & & 2751 \\
\hline $\mathrm{RCH}$ & & 88 & & & 1383 \\
\hline RDTS-GARCH & & 0.0202 & 0.2446 & 0.3742 & 0.0962 \\
\hline & $200^{-}$ & & & & .3261 \\
\hline $\mathrm{ARCH}$ & & 0.0253 & 0.3856 & 312 & 0.1688 \\
\hline RDTS-GARCH & & 0.0227 & 0.3460 & 0.5219 & 0.0944 \\
\hline $\mathrm{CH}$ & 2007 & & & & 3.0362 \\
\hline & & & & & 1.0589 \\
\hline RDTS-GARCH & & 0.0243 & 0.2021 & 0.3101 & 0.5639 \\
\hline $\mathrm{CH}$ & 2007 & & & & 1.6838 \\
\hline & & & & & 0.6683 \\
\hline RDTS-GARCH & & 0.0210 & 0.2051 & 0.3268 & 0.3803 \\
\hline & 20070509 & & & & 979 \\
\hline & & & & & .3702 \\
\hline RDTS-GARCH & & 0.0433 & 0.4380 & 0.6492 & 0.2103 \\
\hline
\end{tabular}




\section{Working Paper Series in Economics}

recent issues

No. 28 Young Shin Kim, Svetlozar T. Rachev, Michele Leonardo Bianchi, Frank J. Fabozzi: Tempered stable and tempered infinitely divisible GARCH models, May 2011

No. 27 Takashi Kanamura, Svetlozar T. Rachev, Frank J. Fabozzi: A profit model for spread trading with an application to energy futures, May 2011

No. 26 Michele Leonardo Bianchi, Svetlozar T. Rachev, Young Shin Kim, Frank J. Fabozzi: Tempered infinitely divisible distributions and processes, May 2011

No. 25 Sebastian Kube, Michel André Maréchal and Clemens Puppe: The currency of reciprocity - gift-exchange in the workplace, April 2011

No. 24 Clemens Puppe and Attila Tasnádi: Axiomatic districting, April 2011

No. 23 Dinko Dimitrov and Clemens Puppe: Non-bossy social classification, April 2011

No. 22 Kim Kaivanto and Eike B. Kroll: Negative recency, randomization device choice, and reduction of compound lotteries, April 2011

No. 21 Antje Schimke and Thomas Brenner: Long-run factors of firm growth - a study of German firms, April 2011

No. 20 Aaron B. Scholz: Spatial network configurations of cargo airlines, April 2011

No. 19 Arne Beck: Public bus transport in Germany - a proposal to improve the current awarding system, April 2011

No. 18 Nina Menz and Ingrid Ott: On the role of general purpose technologies within the Marshall-Jacobs controversy: the case of nanotechnologies, April 2011 